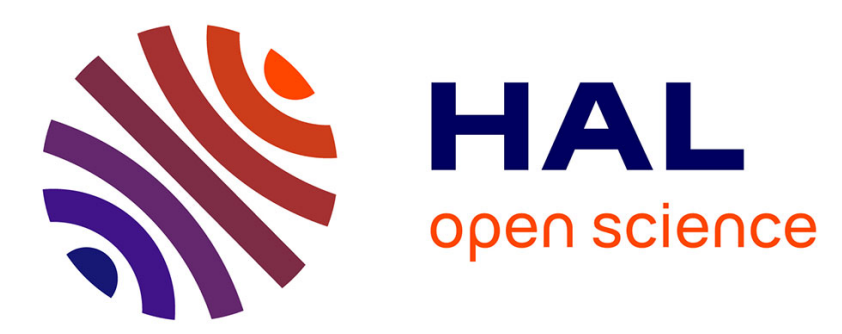

\title{
Three-dimensional non-linear buckling of thick-walled elastic tubes under pressure
}

Yunfei Zhu, Xiaoyu Luo, Huiming Wang, Raymond W. Ogden, Colin Berry

\section{To cite this version:}

Yunfei Zhu, Xiaoyu Luo, Huiming Wang, Raymond W. Ogden, Colin Berry. Three-dimensional nonlinear buckling of thick-walled elastic tubes under pressure. International Journal of Non-Linear Mechanics, 2013, 48, pp.1-14. 10.1016/j.ijnonlinmec.2012.06.013 . hal-01303326

\section{HAL Id: hal-01303326 \\ https://hal.science/hal-01303326}

Submitted on 18 Apr 2016

HAL is a multi-disciplinary open access archive for the deposit and dissemination of scientific research documents, whether they are published or not. The documents may come from teaching and research institutions in France or abroad, or from public or private research centers.
L'archive ouverte pluridisciplinaire HAL, est destinée au dépôt et à la diffusion de documents scientifiques de niveau recherche, publiés ou non, émanant des établissements d'enseignement et de recherche français ou étrangers, des laboratoires publics ou privés. 


\title{
Three-dimensional non-linear buckling of thick-walled elastic tubes under pressure
}

\author{
Y. Zhu ${ }^{\mathrm{a}}$, X.Y. Luo ${ }^{\mathrm{a}}$, H.M. Wang ${ }^{\mathrm{b}}$, R.W. Ogden ${ }^{\mathrm{c}}$, C. Berry ${ }^{\mathrm{d}}$ \\ a School of Mathematics and Statistics, University of Glasgow, Glasgow G12 8QW, UK \\ b School of Aerospace, Xi'an Jiaotong University, PR China \\ ' School of Engineering, University of Aberdeen, King's College, Aberdeen AB24 3UE, UK \\ ${ }^{\mathrm{d}}$ Institute of Cardiovascular and Medical Sciences, BHF Glasgow Cardiovascular Research Centre, University of Glasgow, Glasgow, UK
}

\begin{abstract}
This paper is concerned with numerical simulations of three-dimensional finite deformation of a thick-walled circular elastic tube subject to internal or external pressure and zero displacement on its ends. We formulate the system of equations that can accommodate large strain and displacement for the incompressible isotropic neo-Hookean material. The fully non-linear governing equations are solved using the $\mathrm{C}++$ based object-oriented finite element library libMesh. A Lagrangian mesh is used to discretize the governing equations, and a weighted residual Galerkin method and Newton iteration solver are used in the numerical scheme. To overcome the sensitivity of the fully non-linear system to small changes in the iterations, the analytical form of the Jacobian matrix is derived, which ensures a fast and better numerical convergence than using a numerically approximated Jacobian matrix.

Results are presented for different parameters in terms of wall thickness/radius ratio, and length/radius ratio, as well as internal/external pressure. Validation of the model is achieved by the excellent agreement with the results obtained using the commercial package Abaqus. Comparison is also made with the previous work on axisymmetric version of the same system (Zhu et al., 2008 [34]; Zhu et al. 2010 [43]), and interesting fully threedimensional post-buckling deformations are highlighted. The success of the current approach paves the way for fluid-structure interaction studies with potential application to collapsible tube flows and modeling of complex physiological systems.
\end{abstract}

Non-linear elasticity Finite deformation Large strain, Elastic tubes, Tube buckling

\section{Introduction}

Modeling non-linear finite deformations of cylindrical tubes under pressure is of considerable interest in many engineering and physiological applications. One of the interesting applications is the so-called collapsible tube problem, which focuses on the instability of a finite length tube subject to external pressure, with its two ends constrained on two rigid tubes. Although seemingly simple, this system reveals many rich dynamic features that have stimulated much research in the last 30 years (see, for example $[1-8])$.

Many studies adopt two-dimensional models, which are informative but are limited by the geometrical specialization [9-17]. Three-dimensional models have been developed more recently [18-22], although they have mostly assumed material linearity and the three-dimensional solid mechanics is simplified using thin-shell theories.

Interestingly, bifurcation behavior of the corresponding dry structure, where pressure loading is constant and fluid-structure interaction is absent, is closely tied to the critical stability in collapsible tube flows. It has been found that these different mechanisms (system stability under a static or flow-induced pressure load) lead to similar results except that a substantially higher pressure drop is required to achieve the same level of collapse for the static load case [23]. For this reason, we also briefly review the extensive bifurcation studies that have been conducted on cylindrical tubes and shells.

We mention, in particular, the seminal work of Yamaki [24,25], who investigated the buckling of circular cylindrical thin shells under external pressure for a variety of loadings, boundary conditions and pre-buckling states. Among many interesting observations, he showed that mode 2 bifurcation is the most unstable mode for sufficiently long tubes, and that the most unstable mode number increases as the tube length is decreased. Stability studies concerning geometrically non-linear vibrations and dynamics of circular cylindrical shells, were reviewed by Amabili and Païdoussis [26], with and without fluid-structure interaction. Other recent advances in post-buckling analysis of thin-walled structures were reported by 
Kounadis [27]. With a particular interest in post-buckling behavior Heil and Pedley [23] examined the stability of cylindrical shells under external pressure using a geometrically non-linear shell theory and confirmed that the mode number of the most unstable mode increases as the tube length is decreased, as predicted by Yamaki [25]. There is also an extensive literature on plastic buckling of circular tubes. Experimental and modeling aspects of the compression of steel tubes in the plastic regime have been reviewed in the recent works by Bardi and Kyriakides [28] and Bardi et al. [29]. Additional reviews on thin shell stability are those by Koiter [30,31], Simitses [32], Hutchinson and Koiter [33], and Zhu et al. [34]. In summary, buckling and post-buckling behaviors of thin-walled structures have been studied extensively.

On the other hand, work on post-buckling of thick-walled tubes has not been prominent, although some attempts have been made. Classical or general shell theories were developed on the assumption that lines originally normal to the shell mid-surface remain straight, and that the transverse normal stresses are assumed to be zero. Chróścielewski et al. [35] established values of two correction factors for transverse shear stress resultants and stress couples within the six-field geometrically non-linear theory of elastic shells. They also examined the influence of the theoretical values on the non-linear behavior of regular and irregular shell structures. Since the transverse shear moduli of most laminated shell structures are low, Simitses [32] developed a higher-order shear deformation shell theory by removing several simplifying assumptions in classical shell theory. He found that classical theories are not applicable for moderately thick cylinders, but with a shear correction his theory gives more accurate results. Libai and Simmonds [36] discussed various shell and membrane theories. Plastic localized buckling of moderately thick cylindrical shells under axial compression were examined by Goto and Zhang [37], who observed that the circumferential wave number of the diamond buckling mode increases with a decrease in the wall thickness.

Many experiments have been conducted on collapsible tubes having thick walls [38-41], with a ratio of thickness to inner radius of at least 0.38 , which is significantly thicker than the moderately thick shells considered previously, and cannot be properly described by any existing shell theories. Thick-walled vessels under internal pressure also appear in many other physiological problems, such as left ventricle modeling. It is therefore important to be able to fully assess the three-dimensional mechanical behavior of thick-walled tubes under either internal or external pressure or both. Indeed, very few investigators have studied bifurcations of thick-walled tubes under external pressure with the exceptions of Nowinski and Shahinpoor [42], Marzo et al. [19] and Zhu et al. [34,43], wherein certain model simplifications are adopted.

The present work is a major extension to our previous studies $[34,43]$ in which we presented numerical results for fully non-linear three-dimensional thick-walled cylindrical tubes subject to either internal or external pressure. Bifurcation behavior of thick-walled tubes subject to external pressure and axial loading was examined in Zhu et al. [34] based on the theory of infinitesimal deformations superimposed on a finitely deformed circular cylindrical configuration. Using this theory, the effects of wall thickness and aspect ratio on the initial bifurcation behavior were studied systematically. In Zhu et al. [43], detailed results were given for large axisymmetric deformations of thick-walled cylindrical tubes made of incompressible hyperelastic material subject to zero displacements on their ends and pressure on their external lateral surfaces. It was found that for a thick-walled short tube corner bulging is the typical nonlinear feature. For longer tubes the non-linear model predicts multiple axial modes of deformation.

In the present paper, we extend the axisymmetric analysis to allow for fully three-dimensional deformations. The governing equations, including both geometrical and material non-linearities, are formulated for thick-walled cylindrical tubes of finite length. The material is taken to be incompressible and isotropic and we consider two separate pressure loadings: internal and external pressure on the lateral boundaries of the tube with both ends fixed. The resulting non-linear sets of equations are solved using the object-oriented C++ finite element package libMesh [44], and Abaqus [45]. Since the tube walls collapse suddenly under external pressure, the load-displacement response shows a zero or negative stiffness and strain energy might be released to maintain equilibrium. To trace the equilibrium path for tubes under external pressure, the modified Rik's algorithm $[46,47]$ is used. We consider tubes with different aspect ratios to show the effect of wall thickness and tube length on the non-linear behavior. Corner bulging was found in thick-walled short tubes subject to either internal or external pressure, while the distribution patterns of the shear stresses change significantly. For longer tubes, multiple postbuckling modes are found. For tubes under external pressure the response becomes discontinuous at the onset of buckling, and the critical pressures for each mode have been identified.

\section{Basic equations}

In this section we give a brief summary of the equations governing large elastic deformations with reference to their application to a thick-walled circular cylindrical tube. For a detailed treatment of the non-linear theory, we refer to, for example, Ogden [48].

We consider a circular cylindrical tube with initial geometry corresponding to internal radius $A$, external radius $B$ and length $L$. In the reference configurations we describe points of the tube in terms of the position vector $\mathbf{X}$ relative to an origin situated at the center point of the tube axis. The position vector of the material point $\mathbf{X}$ in the deformed configuration of the tube is denoted by $\mathbf{X}$ and we describe the deformation in the form

$\mathbf{X}=\mathbf{X}+\mathbf{u}$,

where $\mathbf{u}=\mathbf{u}(\mathbf{X})$ is the displacement vector.

We work in terms of rectangular Cartesian coordinates $X_{i}$ and $x_{i}, i=1,2,3$, for the reference and deformed configurations, respectively, with corresponding unit basis vectors $\mathbf{E}_{i}$ and $\mathbf{e}_{i}$. The initial geometry of the tube is then described by

$A \leq \sqrt{X_{1}^{2}+X_{2}^{2}} \leq B, \quad-L / 2 \leq X_{3} \leq L / 2$.

With respect to the chosen bases the deformation gradient tensor $\mathbf{F}$ may be written

$\mathbf{F}=\operatorname{Grad} \mathbf{x}=\frac{\partial x_{i}}{\partial X_{j}} \mathbf{e}_{i} \otimes \mathbf{E}_{j}$,

where summation over indices $i$ and $j$ from 1 to 3 is implied. Under the assumption that the material of the tube is incompressible the constraint

$\operatorname{det} \mathbf{F}=1$

must be satisfied at every material point $\mathbf{X}$.

Let $\mathbf{S}$ denote the nominal stress tensor. For an incompressible hyperelastic material with strain-energy function $W=W(\mathbf{F})$ per unit volume, this is given by

$\mathbf{S}=\frac{\partial W}{\partial \mathbf{F}}-p \mathbf{F}^{-1}$,

where $p$ is a Lagrange multiplier associated with constraint (4). In the absence of body forces the equilibrium equation may then be given in general form as

$\operatorname{Div} \mathbf{S}=\mathbf{0}$, 
where Div is the divergence operator with respect to $\mathbf{X}$. The Cauchy stress tensor $\boldsymbol{\sigma}$ for an incompressible material is related to $\mathbf{S}$ by $\boldsymbol{\sigma}=\mathbf{F S}$, and Eq. (6) may be written equivalently as $\operatorname{div} \boldsymbol{\sigma}=\mathbf{0}$.

For the numerical calculations we use the neo-Hookean strainenergy function, for which $W$ is given by

$W=\frac{1}{2} \mu\left(I_{1}-3\right)$,

where the constant $\mu$ is the shear modulus of the material and $I_{1}$ is defined by $I_{1}=\operatorname{tr}\left(\mathbf{F}^{\mathrm{T}} \mathbf{F}\right)$. From (5) we then have

$\mathbf{S}=\mu \mathbf{F}^{\mathrm{T}}-p \mathbf{F}^{-1}$,

and the corresponding Cauchy stress is $\boldsymbol{\sigma}=\mu \mathbf{F F}^{\mathrm{T}}-p \mathbf{I}$.

We write $\mathbf{u}$ in terms of its components as $\mathbf{u}=u \mathbf{e}_{1}+v \mathbf{e}_{2}+w \mathbf{e}_{3}$. On the ends of the tube we take the displacement to vanish, so that

$u=v=w=0 \quad$ on $X_{3}= \pm L / 2$.

On the lateral surfaces we consider two pressure conditions: for the first case, a pressure $P$, per unit deformed area, is applied on the external lateral surface of the tube while the inner surface is free of traction. For the second case, the pressure is applied on the internal lateral surface and the outer surface is free of traction. Let $\mathbf{M}$ be a unit vector on the reference lateral surface pointing out of the material. Then, $\mathbf{M}=\left(X_{1} \mathbf{E}_{1}+X_{2} \mathbf{E}_{2}\right) / B$ on the outer surface and $\mathbf{M}=-\left(X_{1} \mathbf{E}_{1}+X_{2} \mathbf{E}_{2}\right) / A$ on the inner surface. On the surface where the pressure is specified, denoted $\Gamma_{p}$, the traction boundary condition may then be written

$\mathbf{S}^{\mathrm{T}} \mathbf{M}=-P \mathbf{F}^{-\mathrm{T}} \mathbf{M}$ on $\Gamma_{p}$

where $\mathbf{M}$ is now the appropriate unit normal depending on whether $\Gamma_{p}$ is the inner or outer surface.

\section{Finite element algorithm}

\subsection{Discretization}

The governing PDEs (6) and the constraint Eq. (4) are approximated using the weighted residual Galerkin method, with the elastic domain divided into a set of sub-domains. We have written code based on the open-source finite element library libMesh [44] to solve the non-linear PDEs numerically. We now summarize the details of the discretization and the numerical techniques used. In terms of quadratic shape functions $N_{k}$ and linear shape functions $L_{k}$, the displacement components $(u, v, w)$ and the Lagrange multiplier $p$ are written

$u=\sum_{k=1}^{n_{1}} N_{k}(\xi, \eta) u_{k}, \quad v=\sum_{k=1}^{n_{1}} N_{k}(\xi, \eta) v_{k}$

$w=\sum_{k=1}^{n_{1}} N_{k}(\xi, \eta) w_{k}, \quad p=\sum_{k=1}^{n_{2}} L_{k}(\xi, \eta) p_{k}$,

where $n_{1}$ and $n_{2}$ are the element node numbers, which are dependent on the element type chosen, $\xi$ and $\eta$ are natural coordinate variables, corresponding to isoparametric finite elements, $u_{k}, v_{k}, w_{k}$ are the components of the displacement at node $k$, and $p_{k}$ is the Lagrange multiplier at node $k$.

This allows us to write the discretized non-linear governing Eqs. (6), with (8), and (4) as

$\mathfrak{R} \equiv \mathcal{K}(\mathbf{U}) \mathbf{U}-\mathcal{F}(\mathbf{U})=\mathbf{0}$

where $\mathbf{U}$ is the global vector of unknowns, $\mathcal{K}(\mathbf{U})$ is the global stiffness matrix, $\mathcal{F}(\mathbf{U})$ denotes the force vector, which is also dependent on $\mathbf{U}$, and $\mathfrak{R}$ is the global residual vector, which should be $\mathbf{0}$ for an exact solution. Both tetrahedral and hexagonal elements are used in the simulations. The matrix Eq. (11) is assembled from the finite element matrix equations, which are

$$
\begin{aligned}
& \sum_{j=1}^{n_{1}} \int_{\Omega^{e}} \mu\left(N_{i, 1} N_{j, 1}+N_{i, 2} N_{j, 2}+N_{i, 3} N_{j, 3}\right) \mathrm{d} \Omega^{e} u_{j} \\
& \quad+\sum_{j=1}^{n_{1}} \int_{\Omega^{e}} p\left\{N_{i, 1}\left[-\left(1+w_{, 3}\right) N_{j, 2}+w_{, 2} N_{j, 3}\right]\right. \\
& \left.\quad+N_{i, 2}\left[-w_{, 1} N_{j, 3}+\left(1+w_{, 3}\right) N_{j, 1}\right]+N_{i, 3}\left(-w_{, 2} N_{j, 1}+w_{, 1} N_{j, 2}\right)\right\} \mathrm{d} \Omega^{e} v_{j} \\
& \quad+\sum_{j=1}^{n_{1}} \int_{\Omega^{e}} p\left(-N_{i, 1} N_{j, 3}+N_{i, 3} N_{j, 1}\right) \mathrm{d} \Omega^{e} w_{j} \\
& \quad-\sum_{j=1}^{n_{2}} \int_{\Omega^{e}} N_{i, 1} L_{j} \mathrm{~d} \Omega^{e} p_{j}=-\int_{\Omega^{e}} \mu N_{i, 1} \mathrm{~d} \Omega^{e} \\
& \quad+\oint_{\Gamma^{e}} N_{i}\left(S_{11} M_{1}+S_{21} M_{2}+S_{31} M_{3}\right) \mathrm{d} \Gamma^{e},
\end{aligned}
$$

$$
\begin{aligned}
& \sum_{j=1}^{n_{1}} \int_{\Omega^{e}} p\left\{N_{i, 1}\left[-w_{, 2} N_{j, 3}+\left(1+w_{, 3}\right) N_{j, 2}\right]+N_{i, 2}\left[-\left(1+w_{, 3}\right) N_{j, 1}+w_{, 1} N_{j, 3}\right]\right. \\
& \left.\quad+N_{i, 3}\left(-w_{, 1} N_{j, 2}+w_{, 2} N_{j, 1}\right)\right\} \mathrm{d} \Omega^{e} u_{j} \\
& \quad+\sum_{j=1}^{n_{1}} \int_{\Omega^{e}} \mu\left(N_{i, 1} N_{j, 1}+N_{i, 2} N_{j, 2}+N_{i, 3} N_{j, 3}\right) \mathrm{d} \Omega^{e} v_{j}+\sum_{j=1}^{n_{1}} \int_{\Omega^{e}} p\left(-N_{i, 2} N_{j, 3}\right. \\
& \left.\quad+N_{i, 3} N_{j, 2}\right) \mathrm{d} \Omega^{e} w_{j}-\sum_{j=1}^{n_{2}} \int_{\Omega^{e}} N_{i, 2} L_{j} \mathrm{~d} \Omega^{e} p_{j}=-\int_{\Omega^{e}} \mu N_{i, 2} \mathrm{~d} \Omega^{e} \\
& \quad+\oint_{\Gamma^{e}} N_{i}\left(S_{12} M_{1}+S_{22} M_{2}+S_{32} M_{3}\right) \mathrm{d} \Gamma^{e},
\end{aligned}
$$

$$
\begin{aligned}
& \sum_{j=1}^{n_{1}} \int_{\Omega^{e}} p\left[N_{i, 1}\left(1+v_{, 2}\right) N_{j, 3}-N_{i, 2} v_{, 1} N_{j, 3}-N_{i, 3}\left(1+v_{, 2}\right) N_{j, 1}\right] \mathrm{d} \Omega^{e} u_{j} \\
& \quad+\sum_{j=1}^{n_{1}} \int_{\Omega^{e}} p\left[-N_{i, 1} u_{, 2} N_{j, 3}+N_{i, 2}\left(1+u_{, 1}\right) N_{j, 3}+N_{i, 3}\left(-N_{j, 2}+u_{, 2} N_{j, 1}\right)\right] \mathrm{d} \Omega^{e} v_{j} \\
& \quad+\sum_{j=1}^{n_{1}} \int_{\Omega^{e}} \mu\left(N_{i, 1} N_{j, 1}+N_{i, 2} N_{j, 2}\right. \\
& \left.\quad+N_{i, 3} N_{j, 3}\right) \mathrm{d} \Omega^{e} w_{j}-\sum_{j=1}^{n_{2}} \int_{\Omega^{e}} N_{i, 3} L_{j} \mathrm{~d} \Omega^{e} p_{j}=-\int_{\Omega^{e}} \mu N_{i, 3} \mathrm{~d} \Omega^{e} \\
& \quad+\oint_{\Gamma^{e}} N_{i}\left(S_{13} M_{1}+S_{23} M_{2}+S_{33} M_{3}\right) \mathrm{d} \Gamma^{e}
\end{aligned}
$$

$$
\begin{aligned}
& \sum_{j=1}^{n_{1}} \int_{\Omega^{e}} L_{i}\left[\left(1+v_{, 2}+w_{, 3}+v_{, 2} w_{, 3}-v_{, 3} w_{, 2}\right) N_{j, 1}+\left(v_{, 3} w_{, 1}-v_{, 1}-v_{, 1} w_{, 3}\right) N_{j, 2}\right. \\
& \left.\quad+\left(v_{, 1} w_{, 2}-w_{, 1}-v_{, 2} w_{, 1}\right) N_{j, 3}\right] \mathrm{d} \Omega^{e} u_{j}+\sum_{j=1}^{n_{1}} \int_{\Omega^{e}} L_{i}\left(N_{j, 2}\right. \\
& \left.\quad-w_{, 2} N_{j, 3}+w_{, 3} N_{j, 2}\right) \mathrm{d} \Omega^{e} v_{j}+\sum_{j=1}^{n_{1}} \int_{\Omega^{e}} L_{i} N_{j, 3} \mathrm{~d} \Omega^{e} w_{j}=0
\end{aligned}
$$

where $\Omega^{e}$ is the domain of an element in the reference configuration, $\Gamma^{e}$ is its boundary,,$k$ signifies differentiation with respect to the reference coordinate $X_{k}$ and $M_{1}, M_{2}, M_{3}$ are the components of the unit outward normal to $\Gamma^{e}$.

\subsection{Newton's method and the continuation method}

Eq. (11) is solved in libMesh by employing the SNES library of PETSc [49], which provides a powerful set of numerical routines, including line search and trust region techniques. Newton-Krylov methods constitute the core of the package. The iterative Newton method is used and involves repeated assembly and solution of the equation systems $\mathcal{J} \mathbf{U}=-\mathfrak{R}$, where $\mathcal{J}$ is the true Jacobian 
matrix, which, using (11), is defined by

$\mathcal{J}\left(\mathbf{U}_{r}\right)=\frac{\partial \mathfrak{R}\left(\mathbf{U}_{r}\right)}{\partial \mathbf{U}}=\mathcal{K}\left(\mathbf{U}_{r}\right)+\frac{\partial \mathcal{K}\left(\mathbf{U}_{r}\right)}{\partial \mathbf{U}} \mathbf{U}_{r}-\frac{\partial \mathcal{F}\left(\mathbf{U}_{r}\right)}{\partial \mathbf{U}}$.

Convergence is achieved when the relative residual tolerance $\left\|\mathfrak{R}\left(\mathbf{U}_{r}\right)\right\| /\left\|\mathfrak{R}\left(\mathbf{U}_{0}\right)\right\|$ (in the $\ell 2$ norm) is less than $10^{-8}$, where $\mathfrak{R}\left(\mathbf{U}_{0}\right)$ is the initial residual.

In order to track the post-buckling solutions for tubes under external pressure, we use the modified Riks method [46,47], which takes the load multiplier as an unknown parameter $\lambda$, and solve for it simultaneously with the nodal displacements. In other words, the pressure loading is controlled by

$P=\lambda P_{\text {ref }}$,

where $P_{\text {ref }}$ is the reference load vector. The effective additional arc-length equation is needed to determine $\lambda$, which is given as [47]

$(\Delta \lambda)^{2}+(\Delta \mathbf{U})^{2}=(\Delta l)^{2}$,

where $\Delta \mathbf{U}$ represents the total increment in displacement within the load step, and $\Delta \lambda$ represents the total increment in load multiplier.

As the pressure increases, the tube walls may collapse suddenly where the load-displacement curves exhibit a softening effect. Numerically, the tangent stiffness becomes negative and the classical solution procedures usually fail to converge. The displacement control method enables us to trace the non-linear response through the various buckling modes.

\section{Numerical results}

To demonstrate the effects of the wall thickness and the ratio of length to (external) radius on the non-linear behavior of the tubes, we consider three tube geometries, as in [43]: a thick-walled short tube with $A / B=0.5$ and $L / B=1$; a thick-walled longer tube with $A / B=0.5$ and $L / B=5$, and a thin-walled longer tube with $A / B=0.8$ and $L / B=5$, where $A, B, L$ are the internal radius, external radius, and length of the tube in the reference configuration. All variables presented are made dimensionless, with length scaled by $B$ and stresses scaled by the shear modulus $\mu$, which are both taken to be unity with appropriate and consistent units (i.e. if $B=1 \mathrm{~cm}$, then $\mu=1 \mathrm{dyn} / \mathrm{cm}^{2}$, and if $B=1 \mathrm{~m}$, then $\mu=1 \mathrm{~Pa}$, etc.).

For each tube geometry, we analyze the non-linear deformation separately for internal and external pressures. All simulations are carried out using the libMesh code except for the large values of external pressure, when the numerical convergence becomes extremely difficult to achieve with our existing solver since no pre-conditioners are applied. For the larger pressure values the well-developed commercial package Abaqus is used. Specifically, Figs. 1, 3, 4, and 5 are plotted based on libMesh results, while Figs. 12, 16, and 17 are plotted using the Abaqus results.

In the following, we use $m$ to denote the azimuthal mode number and $n$ the axial mode number. The components of the Cauchy stress $\boldsymbol{\sigma}$ recovered at each node are obtained by including the superconvergent patch recovery process [50], which allows us to trace the variations of the Cauchy stresses at particular element nodes.

\subsection{Internal pressure}

In this section, we present results for tubes subject to internal pressure. For the particular material model we adopt here, only one deformation mode $(m=1, n=1)$ is found, independently of the tube aspect ratio considered.

The solution for the non-linear displacement component $u$ as a function of the internal pressure $P$ is shown as curve 2 in Fig. 1

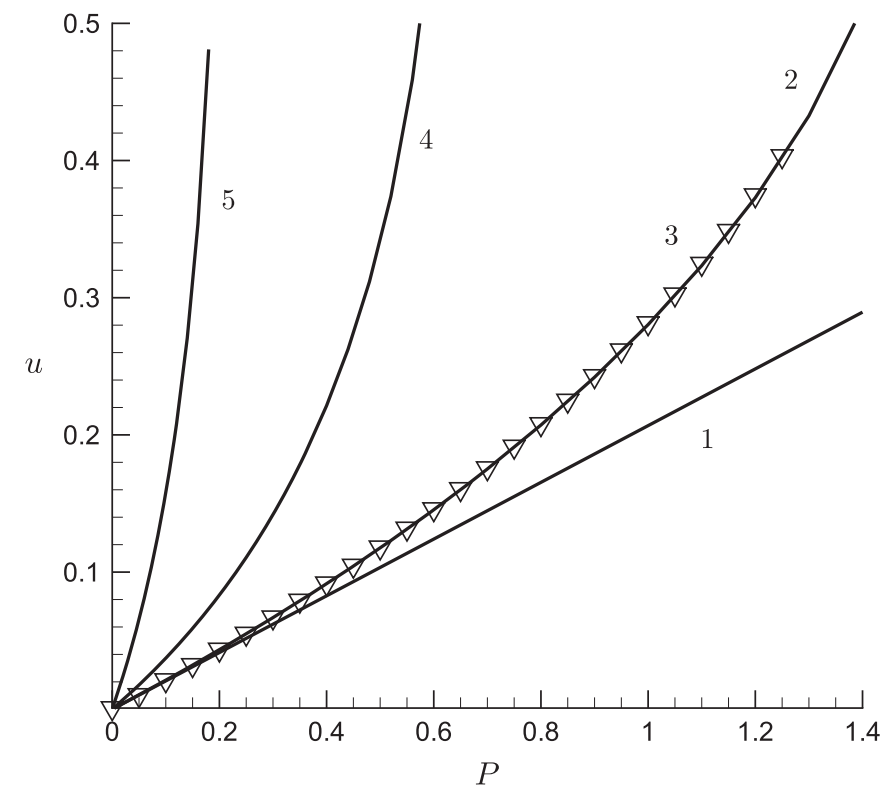

Fig. 1. Displacement component $u$ versus internal pressure $P$ for the thick-walled short tube with $A / B=0.5, L / B=1$ at point $(X, Y, Z)=(0.5,0,0)$ : linear result (curve 1); $3 \mathrm{D}$ non-linear result (curve 2); axisymmetric result (curve 3 with symbols). Curve 4 is for the thick-walled longer tube with $A / B=0.5, L / B=5$ at point $(X, Y, Z)=(0.5,0,0)$, and curve 5 is for a thinner and longer tube with $A / B=$ $0.8, L / B=5$ at point $(X, Y, Z)=(0.8,0,0)$.

plotted at the central point on the inner wall for the tube with $A / B=0.5$ and $L / B=1$. This is compared with the corresponding linear result (curve 1 ) and the axisymmetric non-linear result (curve 3) obtained from the models used in [43]. Fig. 1 shows that the linear and non-linear models agree very well for small values of $P$, but the linear theory underestimates the displacement $u$, especially when $P \gtrsim 0.6$. On the other hand, the axisymmetric and three-dimensional non-linear models (curves 2 and 3 ) are graphically indistinguishable, suggesting that the deformation under internal pressure for $P \lesssim 1.4$ is indeed axisymmetric. Curves 4 and 5 in Fig. 1 are the three-dimensional non-linear results for the longer tubes (also for a central point located on the inner wall). It is not surprising to observe that for the same internal pressure, the deformations of the longer and thinner tubes are much greater. Note that in Fig. 1 and henceforth it is convenient to use the notation $(X, Y, Z)$ instead of $\left(X_{1}, X_{2}, X_{3}\right)$.

For the thick-walled short tube, there is some very small, yet visible, corner (or edge) bulging at the outer wall of the tube as the internal pressure increases to $P=1.5$, as can be seen in Fig. 2 . This is absent in the corresponding linear case (not shown). Corner bulging is also found (but on the inner wall) when the tube is subject to external pressure (see Fig. 8 below) and was previously reported by Zhu et al. [43]. Thus, the non-linear effect of corner bulging can occur under either internal or external pressure. However, the extent of bulging is much smaller for a tube under internal pressure, compared with that for a tube under external pressure. This is because the corner bulging for tubes under internal pressure occurs at the outer wall, where there is more room for the material to expand compared with the situation for corner bulging at the inner wall. We comment that due to the sharp corner singularity, it is not possible to resolve the bulging tip numerically. However, we have checked to make sure our results are mesh independent everywhere else. Note also that the bulging arises from the material inside the tube wall being squeezed out, but the end boundaries of the tube remain fixed.

To illustrate the response of the Cauchy stresses to the internal pressure at a particular location, plots of the stress components $\sigma_{11}, \sigma_{33}, \sigma_{12}$ and $\sigma_{22}$ versus the pressure are shown in Fig. 3, and 
a

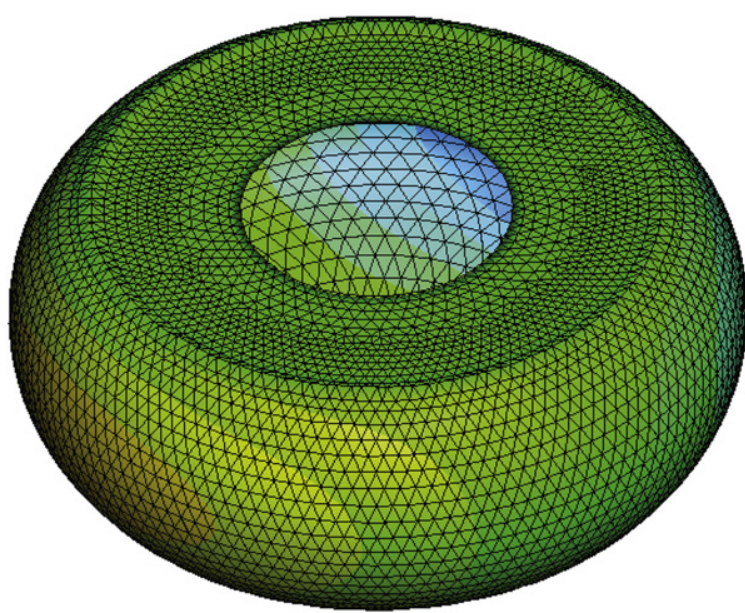

b

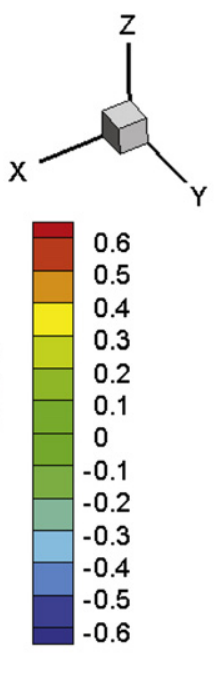

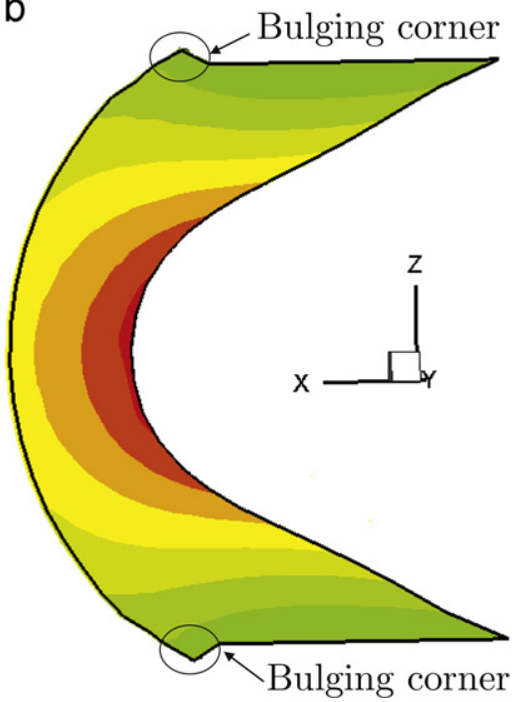

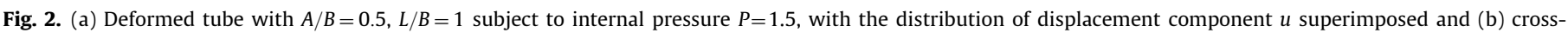
sectional view blow-up showing the corner (edge) bulging.
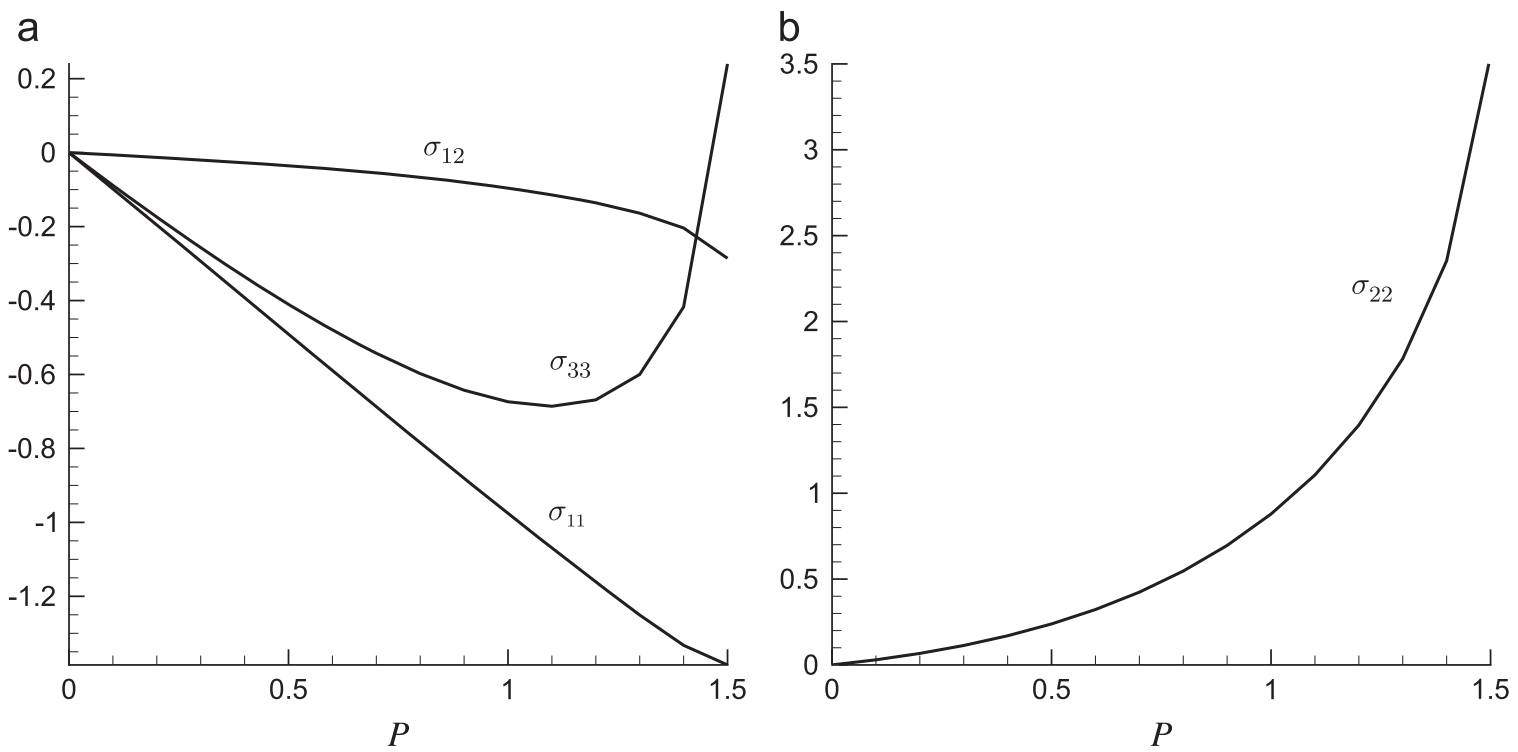

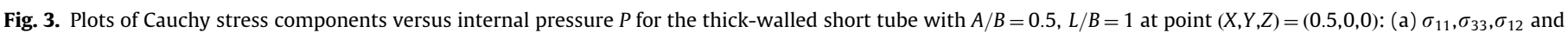
(b) $\sigma_{22}$.

of $\sigma_{11}, \sigma_{33}$ and $\sigma_{22}$ in Figs. 4 and 5. In each case the components not shown are very close to zero. Comparing the three figures, we observe that

1. The magnitude of the normal stress $\sigma_{11}$ increases almost linearly during the deformation process while $\sigma_{22}$ and $\sigma_{33}$ exhibit highly non-linear features; the negative sign of $\sigma_{11}$ indicates that the tube is being compressed in the radial direction; the positive sign of $\sigma_{22}$ is associated with circumferential stretching; the non-monotonic behavior of the axial stress $\sigma_{33}$ is interesting - for small values of $P$ it is negative, corresponding to axial compression, but then becomes positive as the inner radius increases and there is a tendency to extension in the axial direction.

2. For each tube the shear stresses are virtually zero except for very large loading pressures $P ; \sigma_{12}$ is shown to illustrate this, but only in Fig. 3.

3. As the tube become longer and thinner, the magnitude of $\sigma_{11}$ decreases while that of $\sigma_{22}$ increases significantly. For example, at pressure $P=0.15, \sigma_{22}=0.05,0.3,1.3$, respectively, for the tubes with $(A / B=0.5, L / B=1),(A / B=0.5, L / B=5)$ and $(A / B=0.8, L / B=5)$. We have also found that at the center of the inner wall $\sigma_{12}$ is zero for the two longer tubes, but nonzero for the thick-walled short tube. This suggests that the solutions are heavily influenced by the boundary conditions at the ends. Moreover, the turning point of $\sigma_{33}$ in Fig. 3 correlates exactly with the occurrence of corner bulging.

\subsection{External pressure}

Tubes subject to external pressure exhibit many more interesting features than those under internal pressure.

\subsubsection{Thick-walled short tubes: $A / B=0.5$ and $L / B=1$}

If the external pressure is small, the thick-walled short tube deforms essentially axisymmetrically since we have found that the displacement component $v$ is very close to zero (with magnitude of order between $10^{-5}$ and $10^{-3}$ ), and, as shown in 

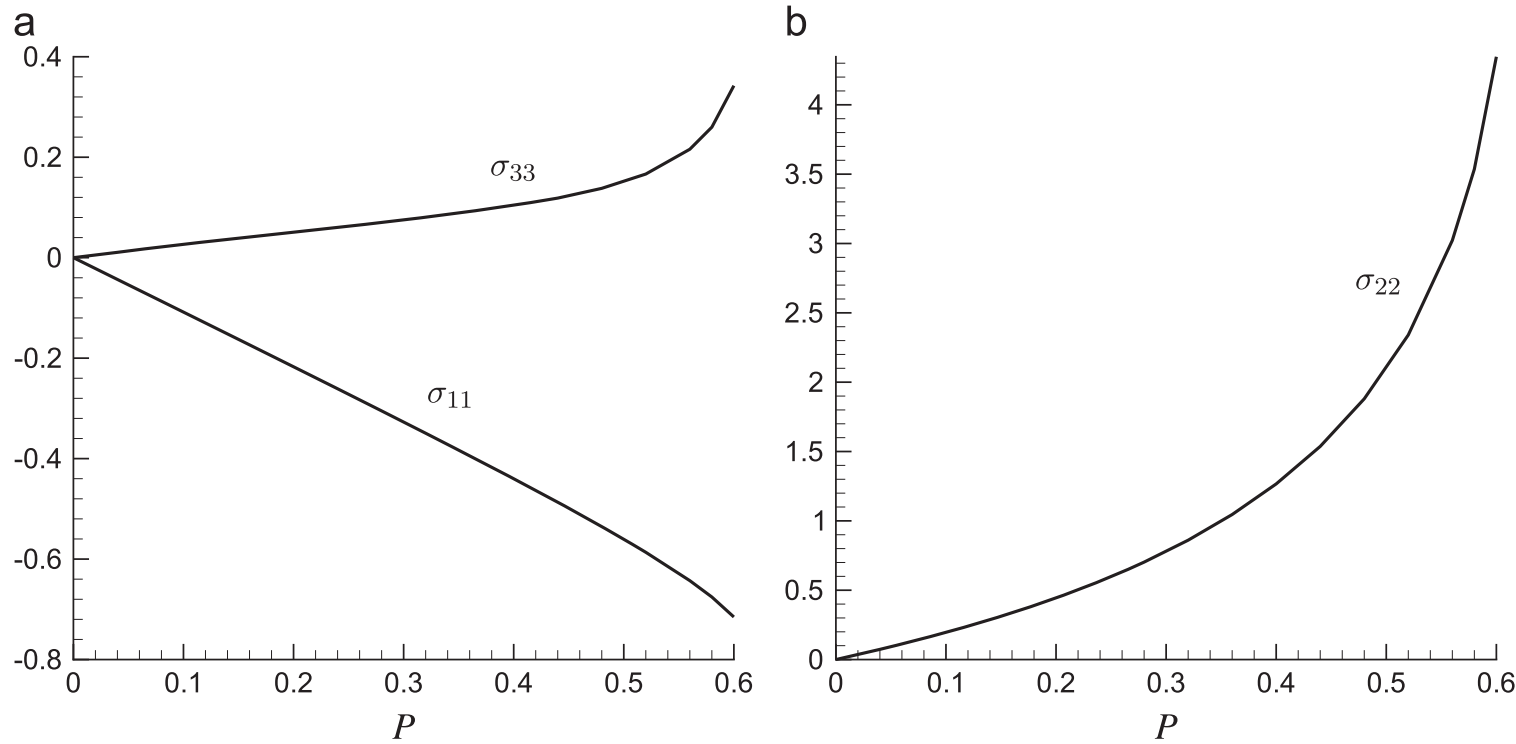

Fig. 4. Plots of Cauchy stress components versus internal pressure $P$ for the thick-walled longer tube with $A / B=0.5, L / B=5$ at point $(X, Y, Z)=(0.5,0,0)$ : (a) $\sigma_{11}, \sigma_{33}$ and (b) $\sigma_{22}$.
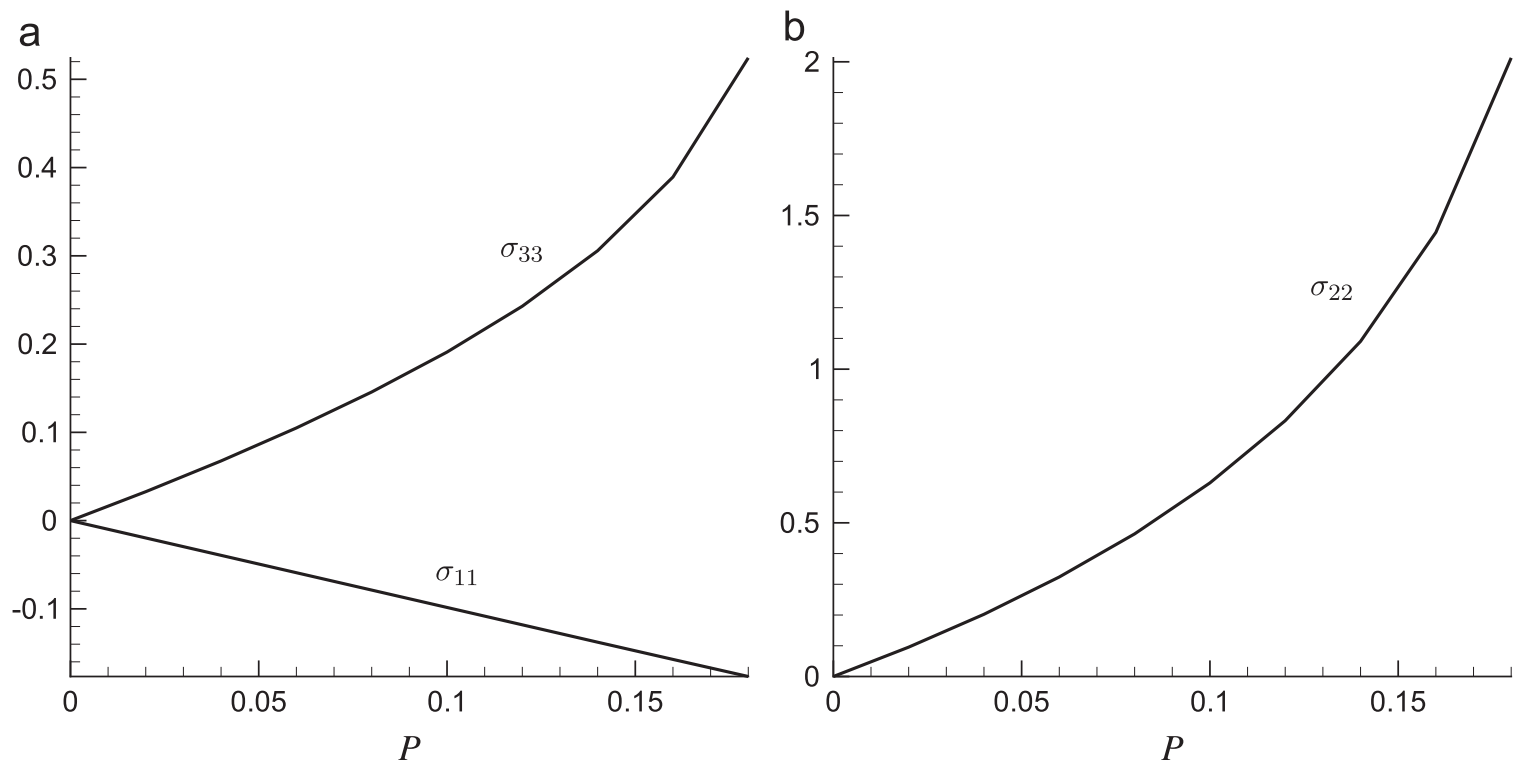

Fig. 5. Plots of Cauchy stress components versus internal pressure $P$ for the thinner and longer tube with $A / B=0.8, L / B=5$ at point $(X, Y, Z)=(0.8,0,0):(a) \sigma_{11}, \sigma_{33}$ and (b) $\sigma_{22}$.

Fig. 6, the displacements $u$ for the three-dimensional and axisymmetric cases are virtually identical. This suggests that prior to bifurcation occurring the deformed thick-walled short tube maintains its axisymmetric shape. This axisymmetric deformation can hold up to $30 \%$ strain, which is well beyond the linear region.

Importantly, the three-dimensional results obtained using our libMesh code agree excellently with those using Abaqus; the detailed displacement and stress distributions from these two numerical approaches are also identical, as illustrated in Figs. 6 and 7. However, we note that Abaqus has more robust solvers, which can handle sudden snap-through buckling much better than the libMesh models developed so far. In the following, we will present results using libMesh for smaller values of external pressure, and Abaqus for large values of the external pressure. Note that in Figs. 7-9 the plot ranges of values have been chosen so as to distinguish the different contours. We remark that in Fig. 7(b), the maximum value of the stress $\sigma_{12}$ at $Z=0$ is computed to be the same $(=0.23)$ using libMesh and Abaqus.
Fig. 8 shows the displacement $u$ and shear stress $\sigma_{13}$ distributions for $P=2.0$ for the cross-section at $Y=0$. This is the case when the inner wall bulges out at the corners. Similar corner bulging behavior for the axisymmetric case was discussed in detail in [43]. Qualitatively, the patterns of shear stresses due to corner bulging (i.e. shear splitting with alternating signs in different regions) is captured again by the general three-dimensional theory. Because of the corner bulging, the distributions of the shear stresses $\sigma_{12}, \sigma_{13}, \sigma_{23}$ in the cross-section at $Z=0.35$ are complex, as shown in Fig. 9, where four sub-zones are found in which $\sigma_{12}$ changes sign. The peak shear stress $\sigma_{12}$ (positive and negative) occurs at the internal wall of the tube.

As in Section 4.1, we trace the variations of the stresses $\sigma_{11}, \sigma_{12}$ $\sigma_{22}$ and $\sigma_{33}$ versus the pressure $P$ near the point $(X, Y, Z)=$ $(-0.5,0,0)$. These results are shown in Fig. 10. The stress components that are close to zero are not included. Fig. 10(b) shows that the most significant load bearing stress component is $\sigma_{22}$, which is ten times greater in magnitude than the other stress components and 
decreases almost linearly with increasing $P$. Hence the tube is predominantly under compression. The other stress components are smaller in magnitude but exhibit non-linear behavior, particularly in the case of $\sigma_{33}$, which has a similar pattern to that in the case of internal pressure loading; it decreases first with the increase of $P$ but then increases when $P$ is greater than about 1.2. Again, this coincides with the appearance of the corner bulging. Different

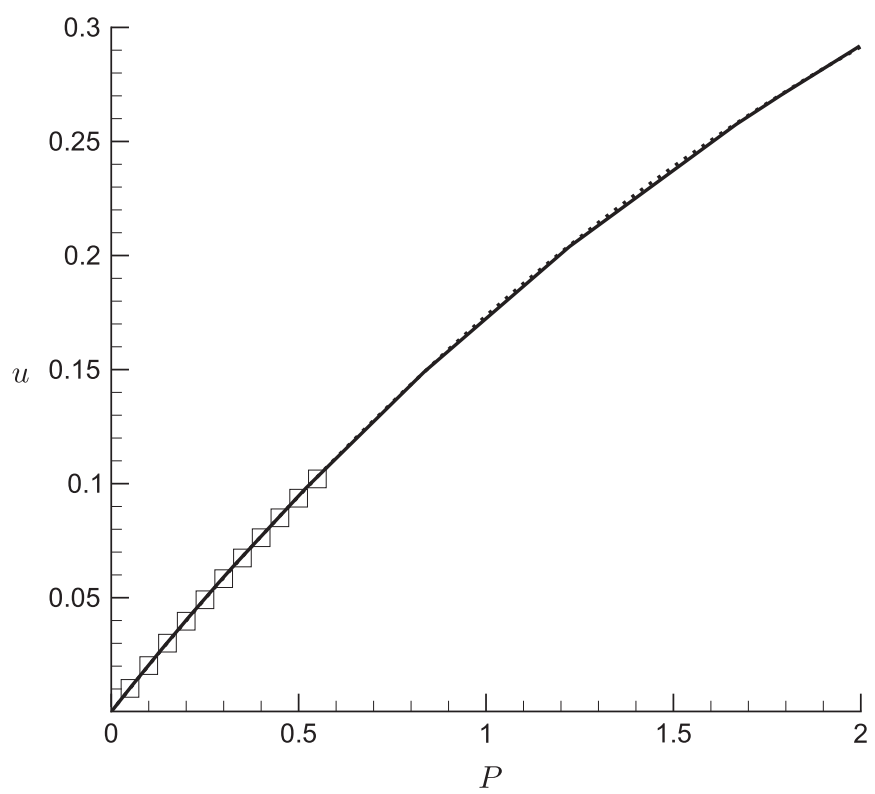

Fig. 6. Displacement component $u$ versus external pressure $P$ for the thick-walled short tube $(A / B=0.5, L / B=1)$ at point $(X, Y, Z)=(-0.5,0,0)$; axisymmetric result (dotted curve); Abaqus result (solid curve); libMesh result (symbols). boundary conditions have also been tested and we find that the bulging corner emerges as a result of the material non-linearity and boundary conditions. There is no bulging effect in the linear case with the same boundary conditions.

\subsubsection{Thick-walled longer tubes: $A / B=0.5$ and $L / B=5$}

For longer tubes, higher modes of deformation occur as the external pressure increases. Fig. 11 shows three different deformation patterns for the thick-walled longer tube with $A / B=0.5$ and $L / B=5$, which correspond to mode numbers $(m=2, n=1)$, $(m=2, n=2)$, and $(m=3, n=1)$. Clearly, these are no longer axisymmetric deformations.

The bifurcation observed here is quite different from the analytical prediction of Zhu et al. [34] (see Fig. 12 therein), where, using three-dimensional incremental equilibrium equations, they predict that only mode ( $m=2, n=1)$ bifurcation may occur from a deformed circular cylindrical configuration for the given parameters. The fundamental difference in this study is that the tube configuration is not circular cylindrical prior to bifurcation. In fact, almost all other published works concerned with tube bifurcation analysis have adopted the assumption that the initial configuration is circular cylindrical. The different bifurcation patterns predicted here highlight the importance of using a fully three-dimensional approach for predicting bifurcations of tubes under large deformations.

On the other hand, for the axial modes, our previous findings in [43] still hold, i.e. longer tubes favor mode $n=2$ deformations, while shorter tubes prefer mode $n=1$, as illustrated in Fig. 11 .

The various bifurcation routes can be better viewed from the pressure-displacement plot, shown in Fig. 12. Initially the solution is unique and the cross-section remains circular when the external pressure is increased. Then, a first bifurcation occurs at about $P=0.27$ (point $\mathbf{a}$ ) when one of the solution branches jumps a

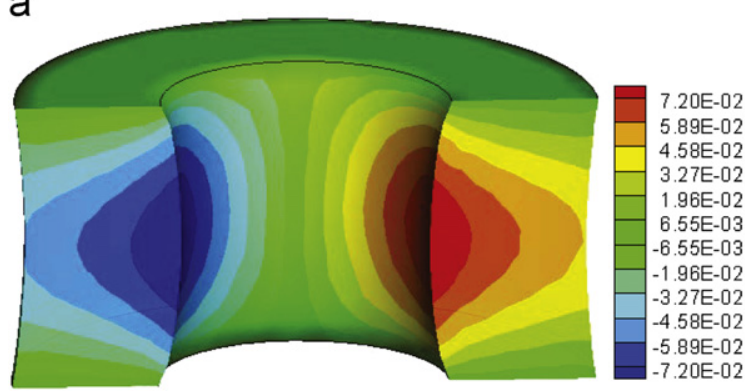

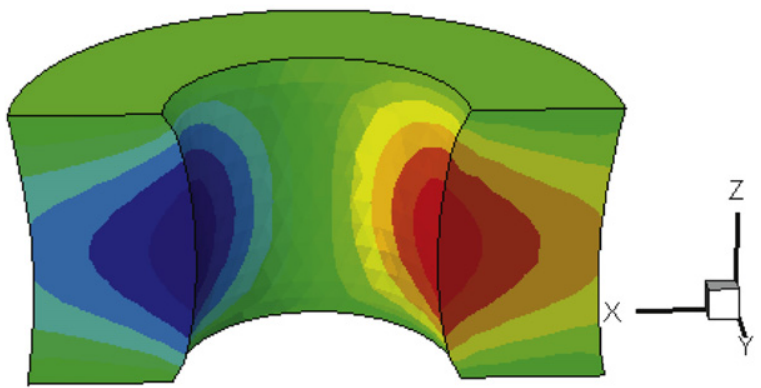

b

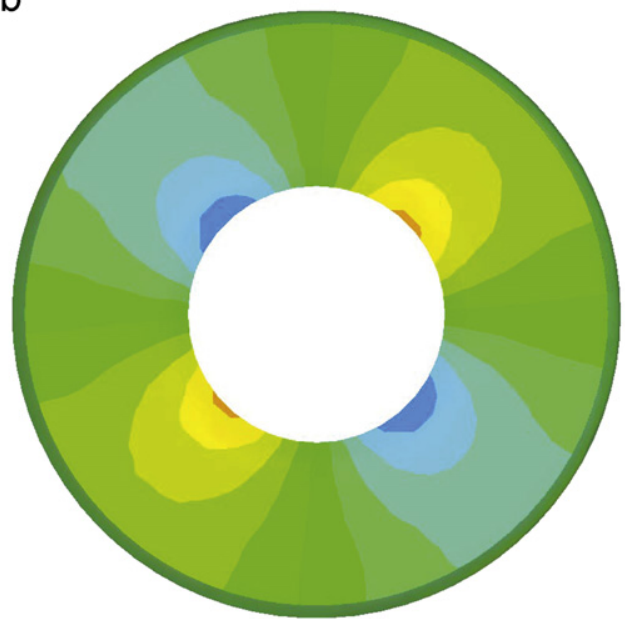

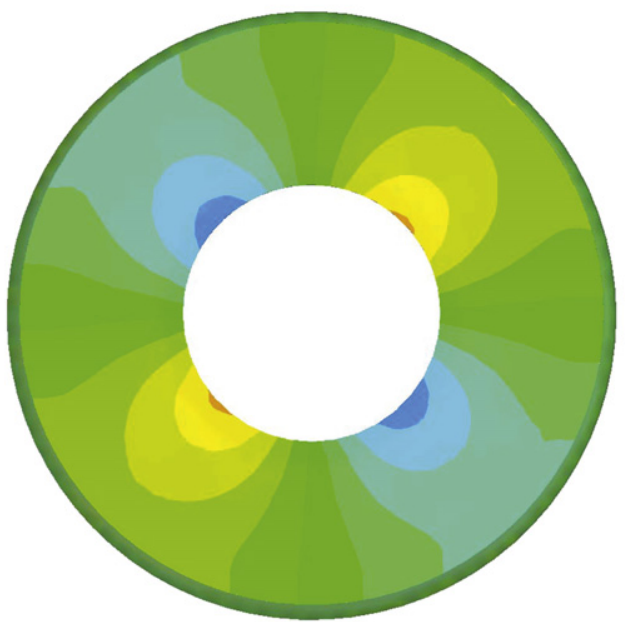

3.38E-01

2.84E-01

2.30E-01

1.76E-01

1.22E-01

6.81E-02

1.42E-02

$-3.98 \mathrm{E}-02$

$-9.38 \mathrm{E}-02$

$-1.48 \mathrm{E}-01$

$-2.02 \mathrm{E}-01$

$-2.56 \mathrm{E}-01$

$-3.10 \mathrm{E}-01$

Fig. 7. Comparison of results from Abaqus (left) and libMesh (right) for external pressure $P=0.4$ : (a) side view of $u$ at $Y=0$ and (b) top view of $\sigma_{12}$ at $Z=0$. 
a

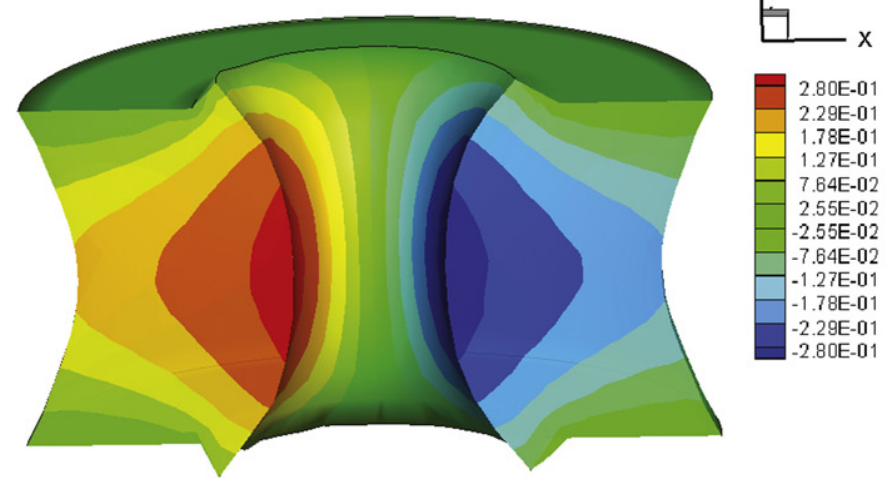

b

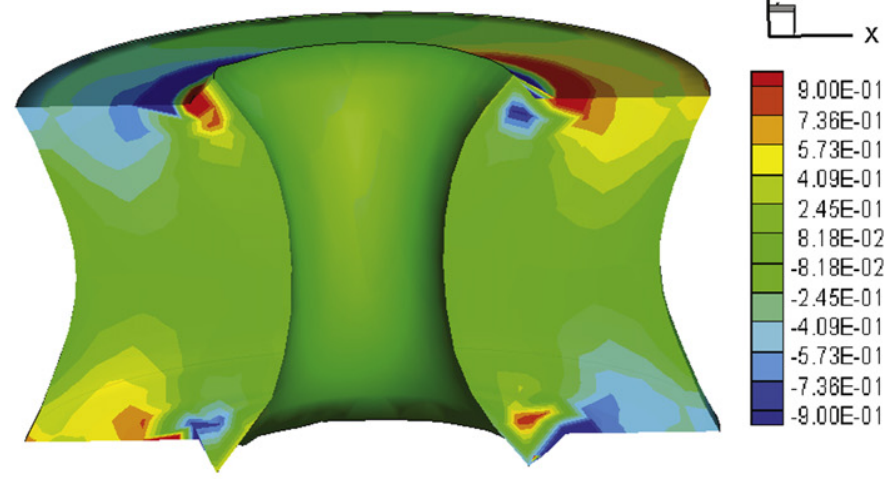

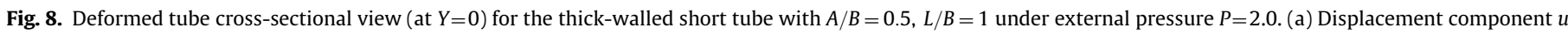
and (b) shear stress $\sigma_{13}$.

a

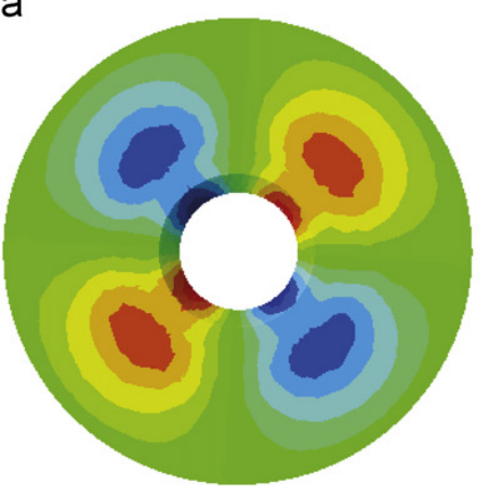

b

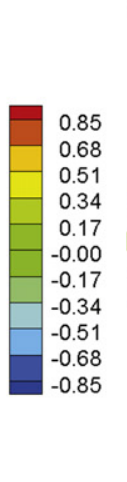

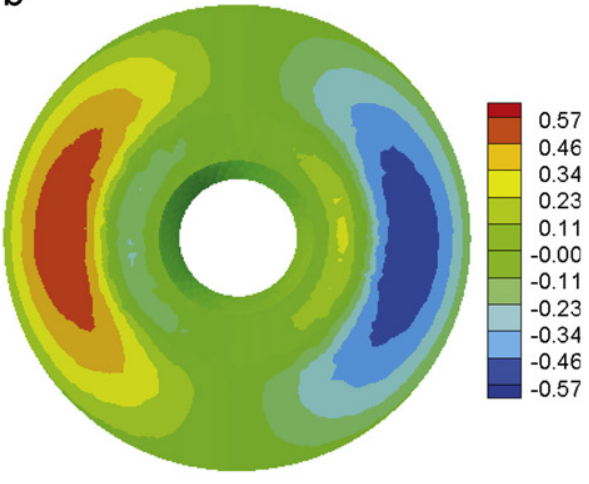

C

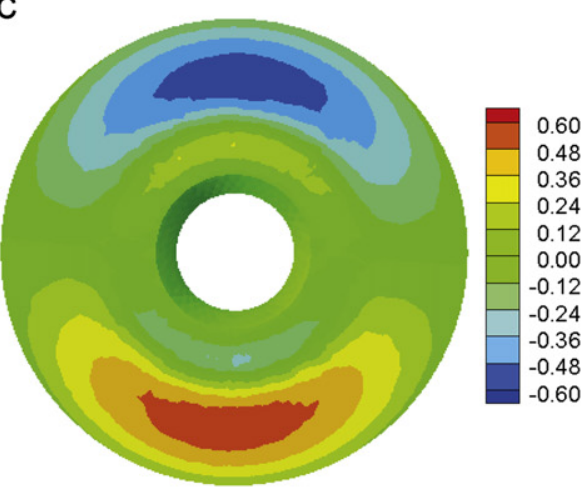

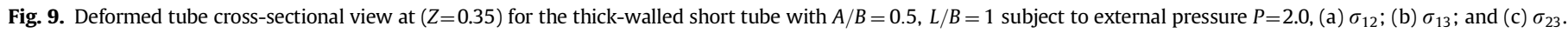

a

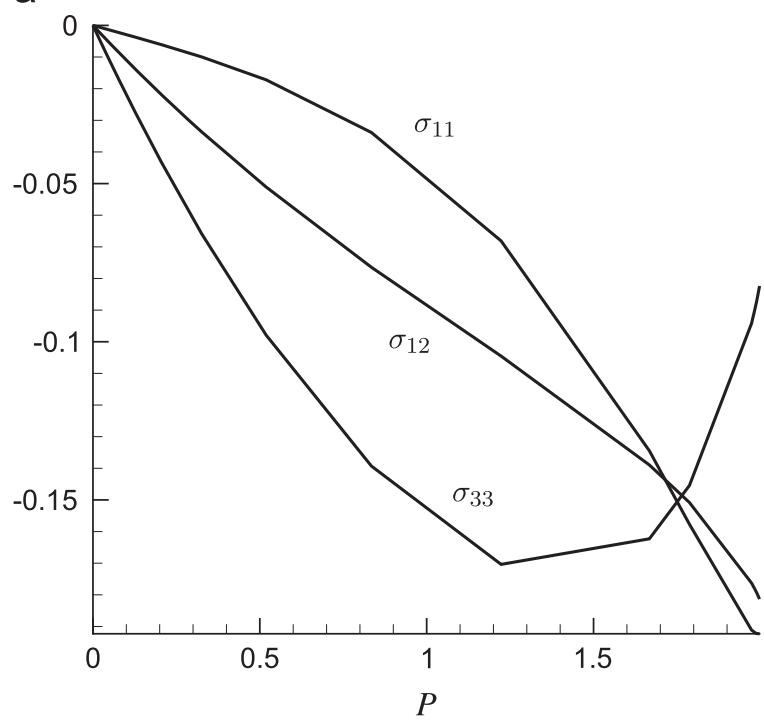

b

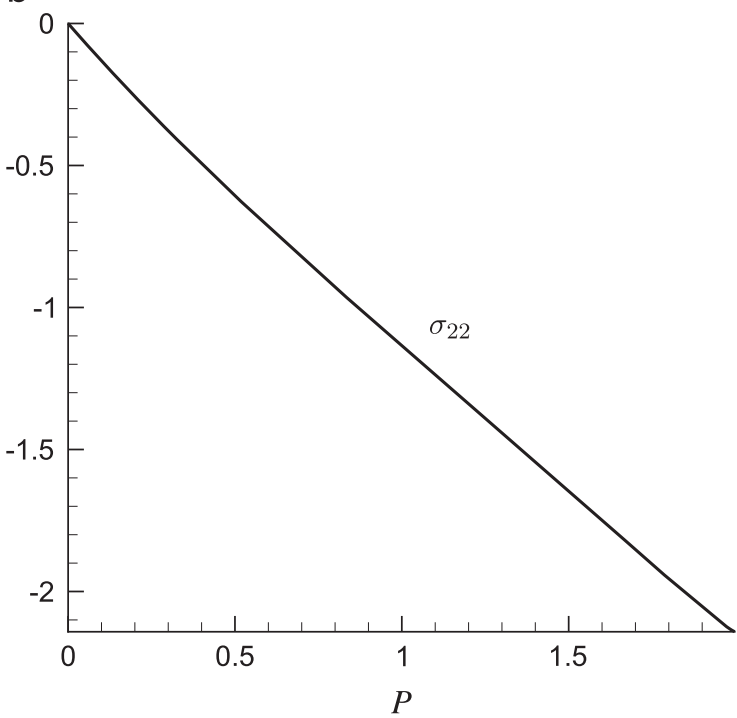

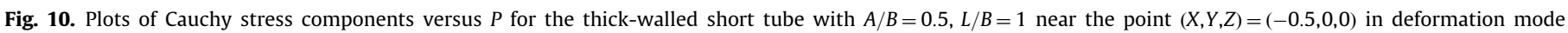
$(m=1, n=1)$ : (a) $\sigma_{11}, \sigma_{33}, \sigma_{12}$ and (b) $\sigma_{22}$.

to point d then follows the post-buckling path 1 (mode $m=2$, $n=1$ ). Another solution branch remains unbuckled until a greater pressure is applied, but then there is a snap-through from points b to e, followed by the post-buckling path 2 (mode $m=2, n=2$ ).
The third solution branch buckles at slightly higher pressure (point c) and then jumps into the post-buckling mode $(m=3$, $n=1)$ at $\mathbf{f}$. These post-buckling modes are clearly shown in Fig. 11. Note that all the circumferential modes we refer to here are for 
a
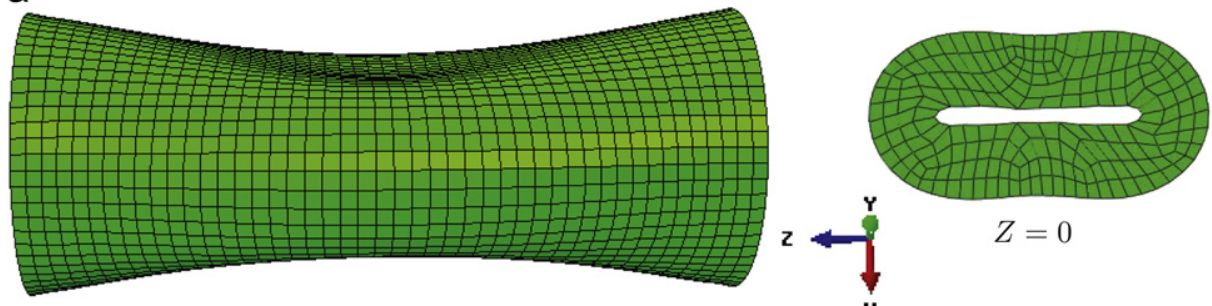

b
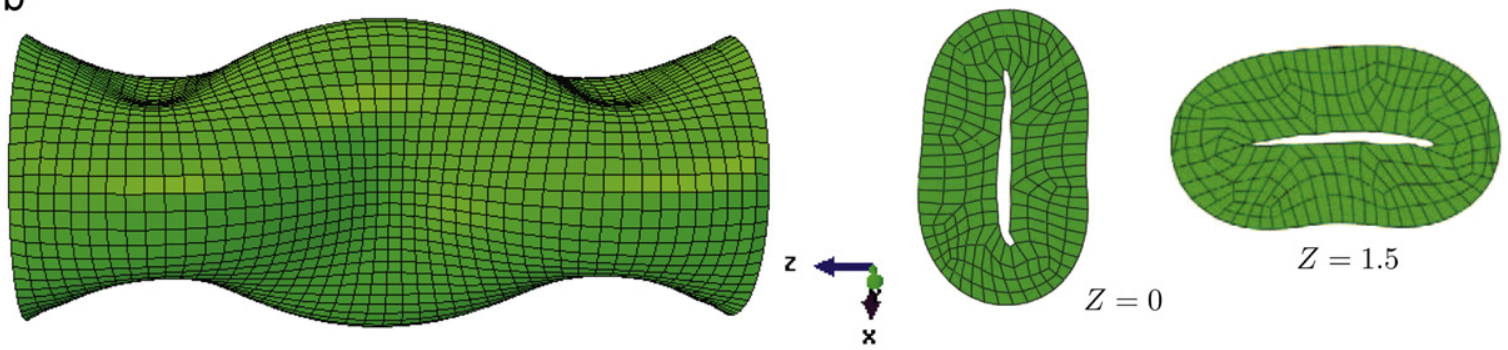

C
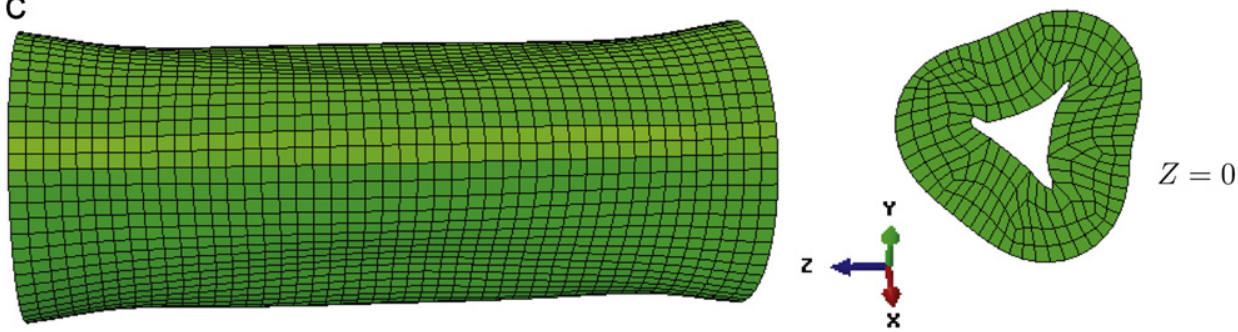

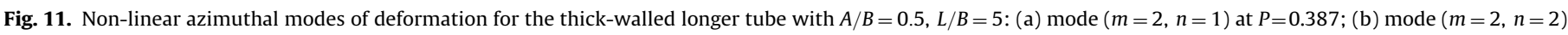
at $P=0.840$; and (c) mode $(m=3, n=1)$ at $P=1.12$. Cross-sectional cuts at $Z=0$ and $Z=1.5$ are shown on the right.

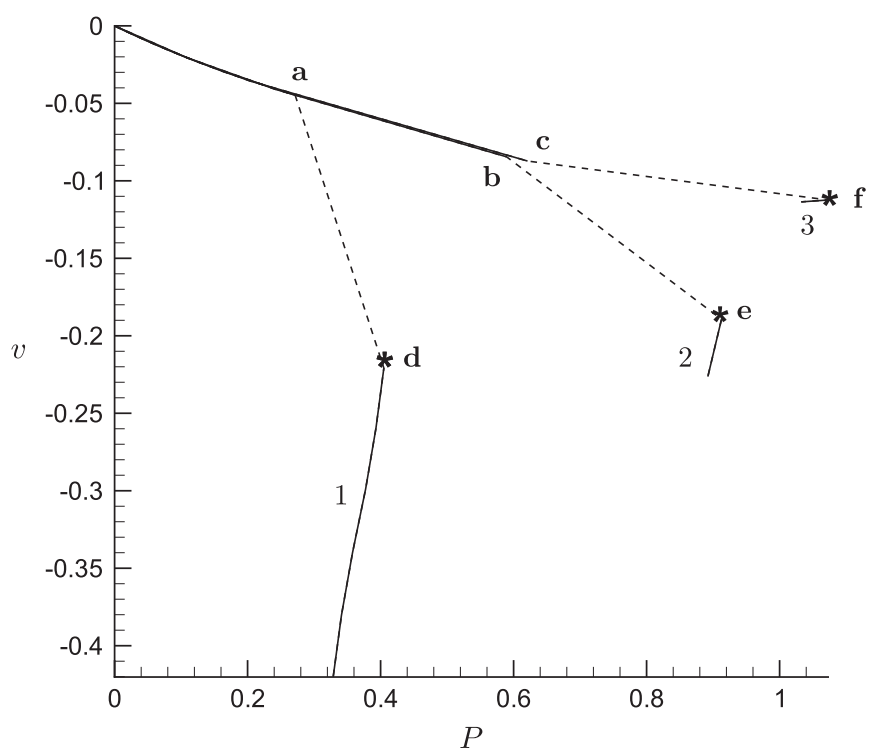

Fig. 12. Displacement component $v$ versus external pressure $P$ for the thickwalled longer tube $(A / B=0.5, L / B=5)$ at point $(X, Y, Z)=(-0.4,0.3,-0.1)$. The solution bifurcates at points $\mathbf{a}, \mathbf{b}, \mathbf{c}$ and buckles into mode $(m=2, n=1)$ following path 1 , mode $(m=2, n=2)$ following path 2 , and mode $(m=3, n=1)$ following path 3.

the central cross-section of the tube $(Z=0)$, since mode-2 (elliptical cross-sectional shape) can occur before $\mathbf{d}$ at off-center locations, e.g. when $Z= \pm 1.5$. To follow these snap-through bifurcations, very small arc-length increments have to be used to trace the solution paths. Clearly, all these are sub-critical bifurcations.
4.2.3. Thinner and longer tubes: $A / B=0.8$ and $L / B=5$

For the thinner and longer tubes with $A / B=0.8 L / B=5$, the deformations are quite complex. A total of eight more post-buckling modes are found in our calculations. These are $(m=1, n=2),(m=$ $2, n=1),(m=2, n=2),(m=3, n=1),(m=4, n=1),(m=4, n=2)$, $(m=4, n=3)$ and $(m=5, n=1)$. These higher modes exhibit fascinating patterns of deformations, as shown in Figs. 13-15, with part of the tube strongly collapsed.

Fig. 16(a) demonstrates three of the higher-mode bifurcations ( $m=1,3,5, n=1)$. Initially, the tube cross-section at $(Z=0)$ remains circular only up to a relatively small external pressure, $P \lesssim 0.018$. Then one solution branch bifurcates into the post-buckling mode $(m=2, n=1)$ at point $\mathbf{a}$, and the magnitude of $v$ increases sharply along path 1 . The second solution branch bifurcates at $\mathbf{b}$ when $P \simeq 0.06$, following the post-buckling mode $(m=3, n=1)$ along path 2. The third solution branch continues to resist buckling until a greater pressure $P \simeq 0.285$ is reached, and then bifurcates into mode $(m=5, n=1)$ along path 3 . Although the bifurcation pattern is similar to that for the thick-walled longer tube shown in Fig. 12, there is no obvious jump along the solution paths, although the displacement changes significantly after the bifurcations. The second and third post-buckling modes are shown in detail in Fig. 14.

A different solution path can be viewed in Fig. 16(b), where the displacement $u$ is plotted against $P$. As the tube buckles from mode $(m=2, n=1)$ into mode $(m=2, n=2)$ at point $\mathbf{a}, u$ jumps from 0.063 to 0.22 . Then it changes to mode $(m=4, n=1)$ at point b with $u$ dropping almost to zero. At point $\mathbf{c}$, however, the tube transitions back into mode $(m=2, n=2)$, which is maintained through point $\mathbf{d}$, but with the azimuthal mode rotating around the $Z$-axis by 90 degrees, as Fig. 13 illustrates.

The solution paths for the other higher modes $(m=4$, $n=1,2,3$ ) are shown in Fig. 17. Here the bifurcations occur at 

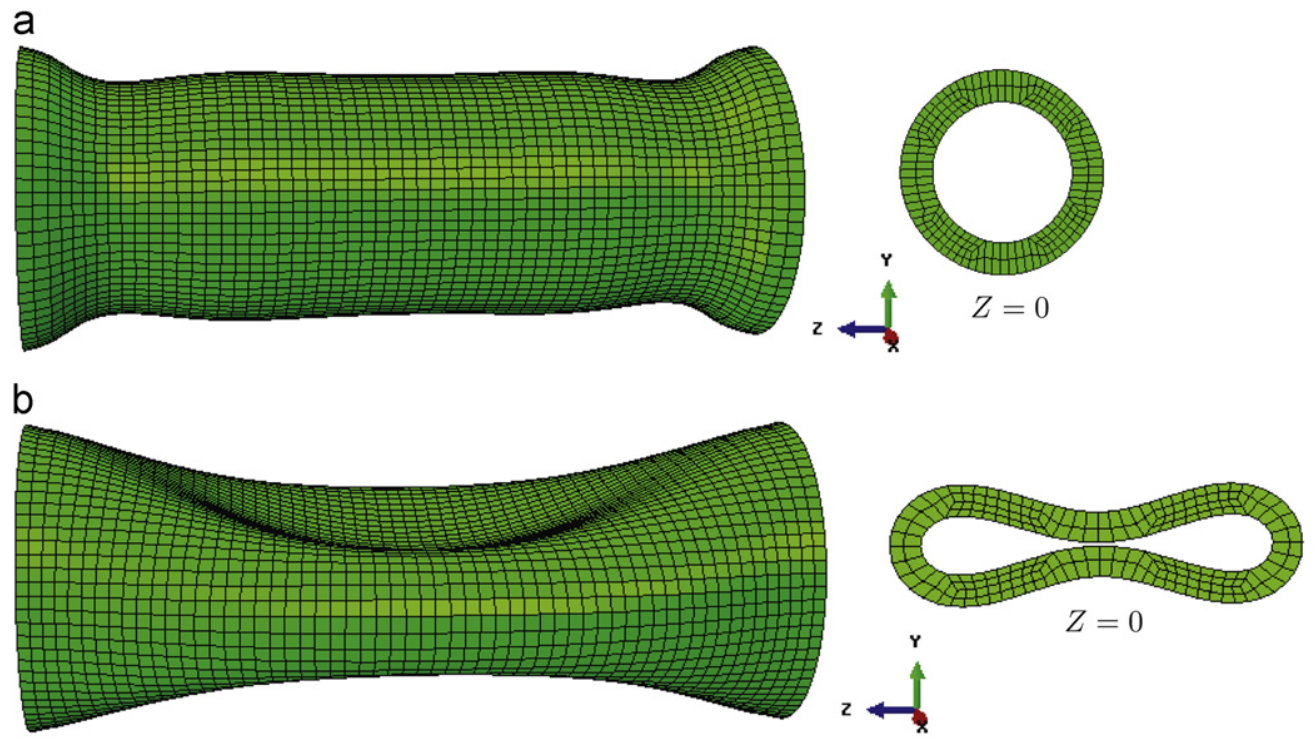

C
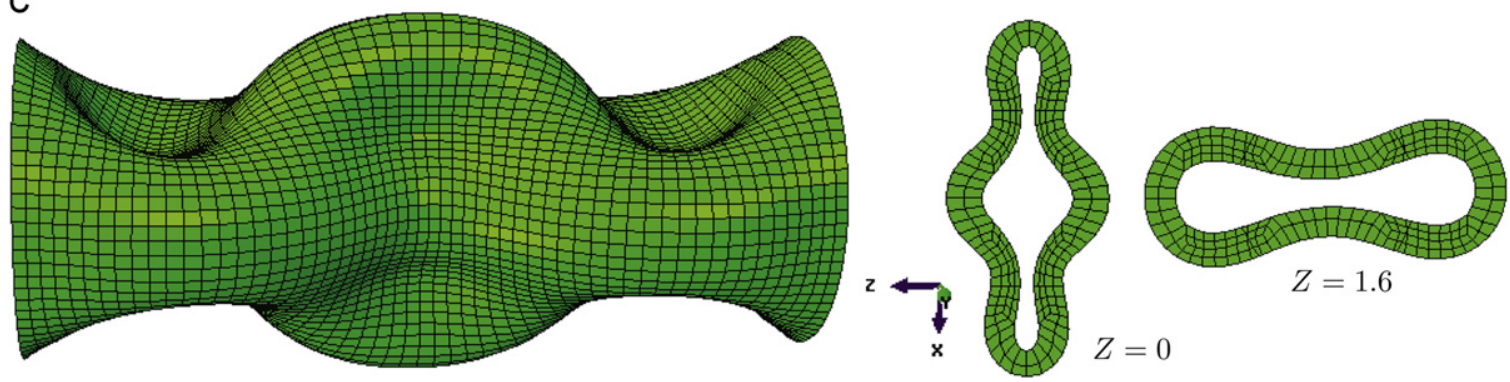

Fig. 13. Non-linear azimuthal modes of deformation for the thinner and longer tube with $A / B=0.8, L / B=5$ : (a) mode $(m=1, n=2)$ at $P=0.389$; (b) mode ( $m=2, n=1$ ) at $P=0.0186$; (c) mode ( $m=2, n=2)$ at $P=0.129$. Cross-sectional cuts at $Z=0$ and $Z=1.6$ are shown on the right.

a
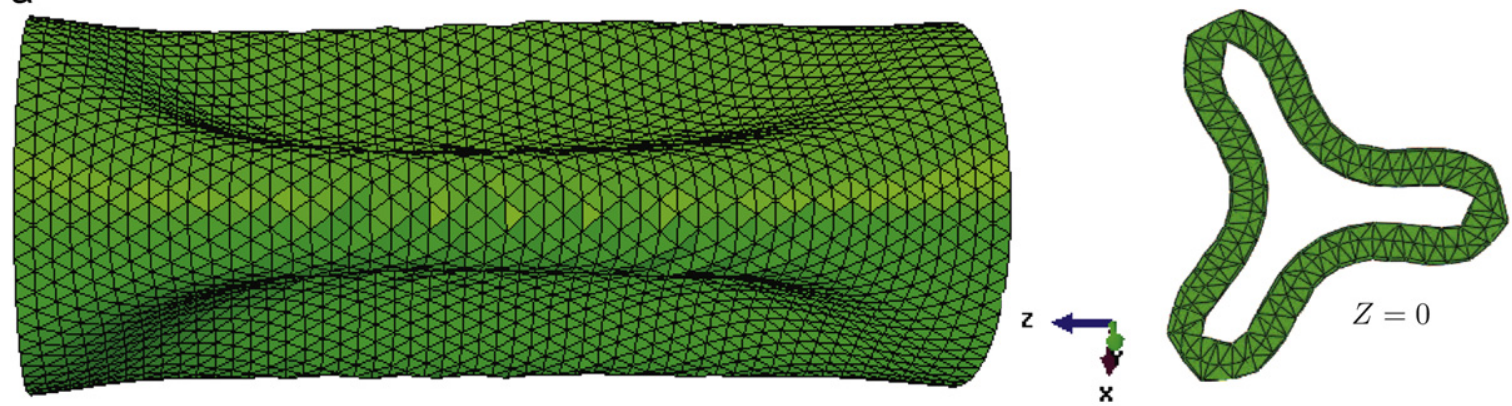

b
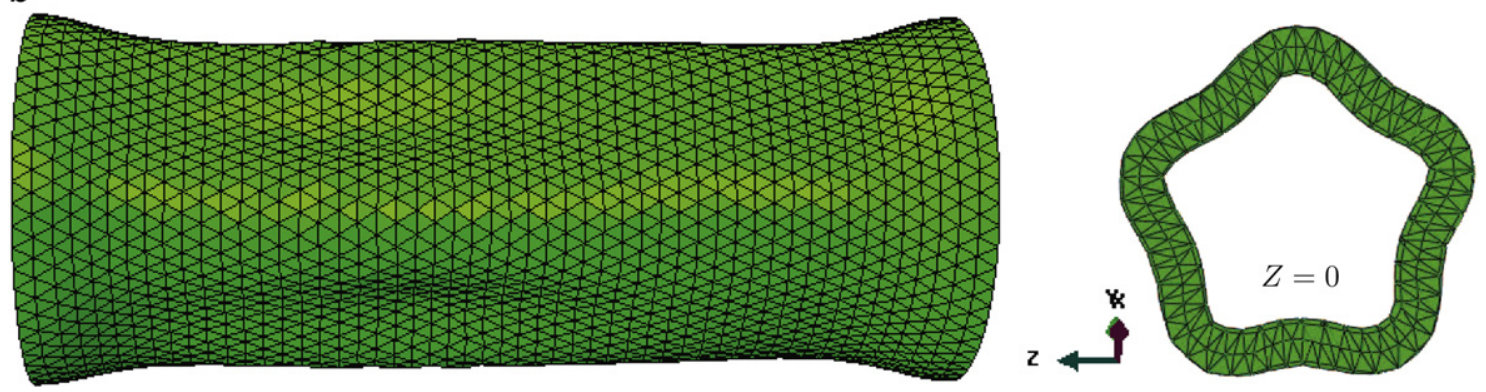

Fig. 14. Non-linear axial modes of deformation for the thinner and longer tube with $A / B=0.8, L / B=5$ : (a) mode $(m=3, n=1)$ at $P=0.082$ and (b) mode ( $m=5, n=1)$ at $P=0.271$. Cross-sectional cuts at $Z=0$ are shown on the right.

$P \simeq 0.08$ (point a), $P \simeq 0.09$ (point $\mathbf{b}$ ), and $P \simeq 0.16$ (point $\mathbf{c}$ ), respectively, into the higher modes $(m=4, n=1,2,3)$. The bifurcation patterns are similar to those shown in Fig. 16(a), although the post-buckling paths 2 and 3 are less steep. Again, there are no obvious jumps in these solution branches. The post-buckling modes are shown in Fig. 15. 
a

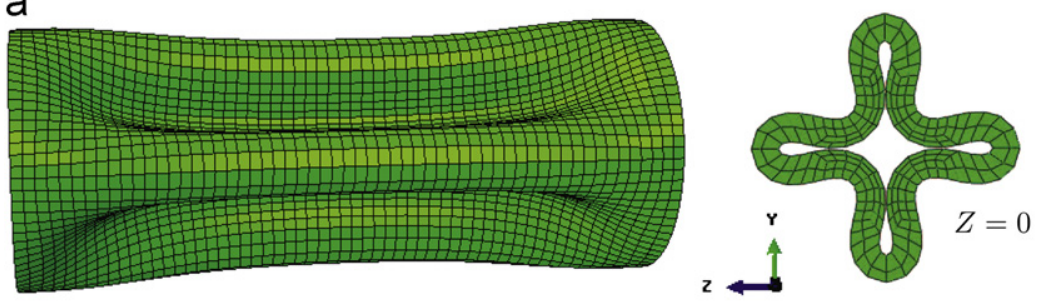

b

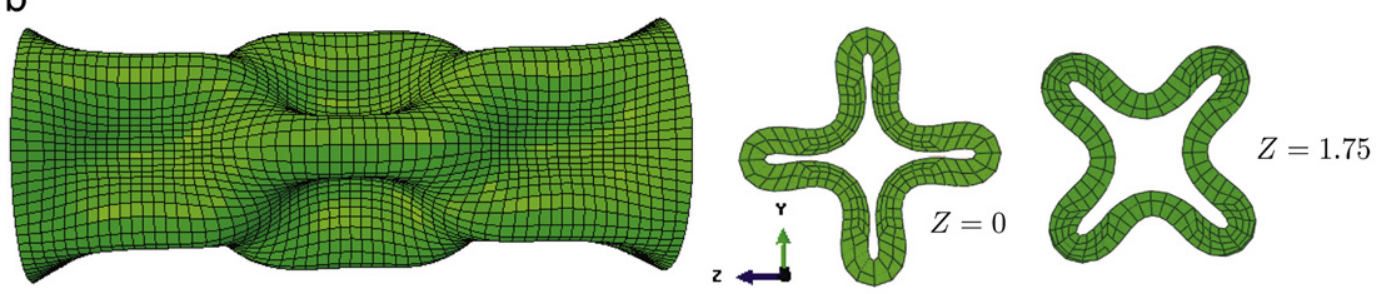

C
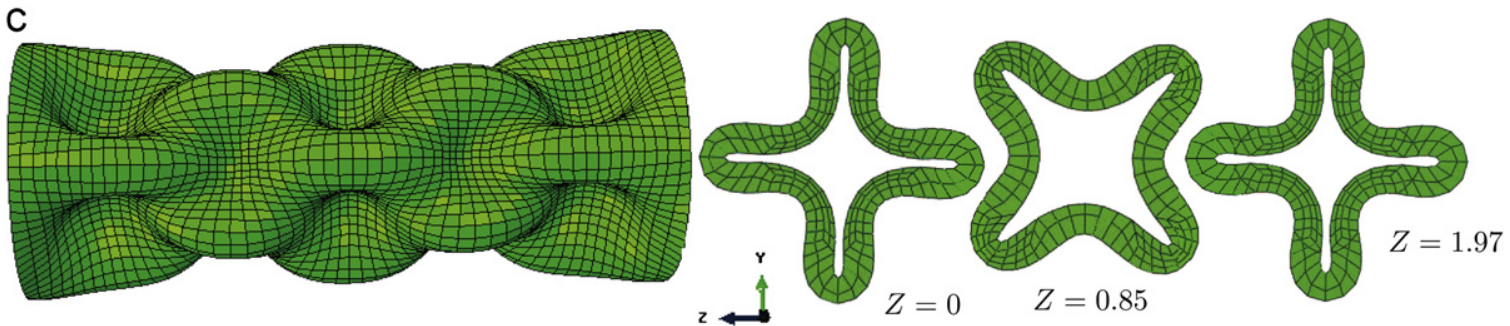

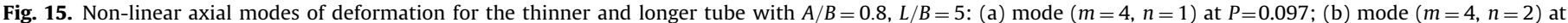
$P=0.158$; and (c) mode $(m=4, n=3)$ at $P=0.251$. Cross-sectional cuts at $Z=0,0.85,1.75$ and 1.97 are shown on the right.

a

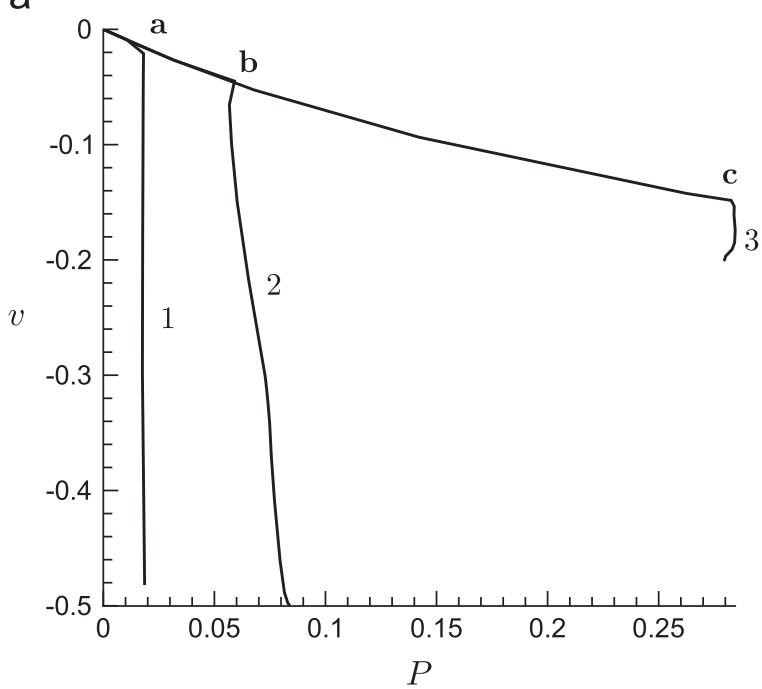

b

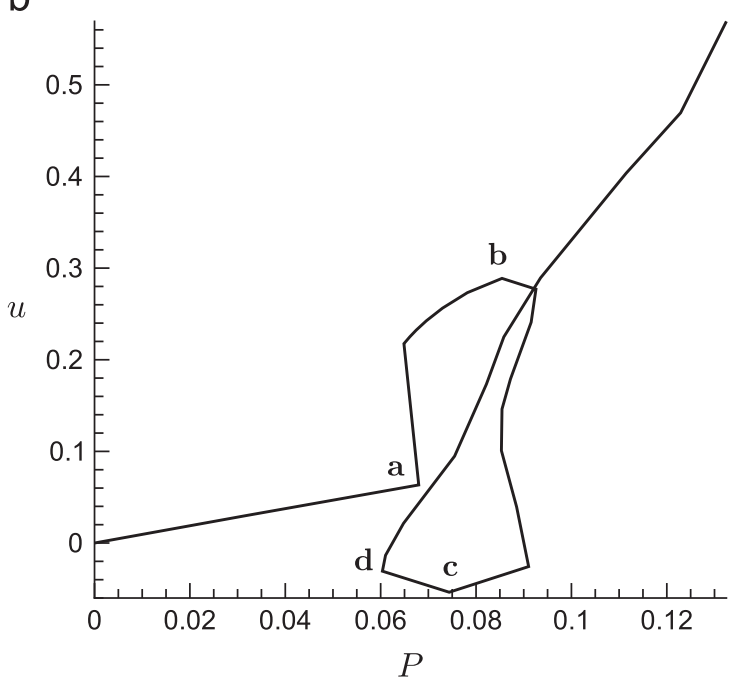

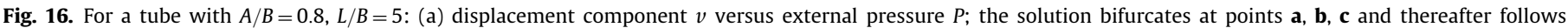

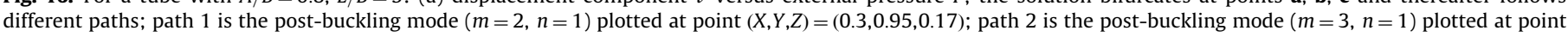

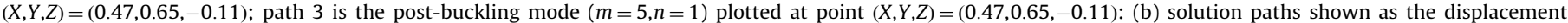
component $u$ versus $P$ plotted at point $(X, Y, Z)=(-0.7,0.4,1.64)$.

Finally, we remark that the solution branches are also very sensitive to geometric imperfections and hence grid asymmetries. The results for modes $(m=3, n=1)$ and $(m=5, n=1)$ are obtained using tetrahedral elements, Fig. 14. All the other modes shown in Figs. 13 and 15 are computed with hexagonal elements. The reason for using different elements is that the onset of postbuckling solutions is highly sensitive to initial perturbations. Small perturbations in the mesh geometry may lead the equilibrium paths into different branches. Therefore, using different types of elements, we can follow certain solution branches much more easily. For example, modes 3 and 5 only occur if we use tetrahedral meshes.

\section{Discussion and conclusions}

The general three-dimensional fully non-linear equations of equilibrium in Lagrangian form have been solved numerically in order to investigate the interesting post-buckling behavior of 


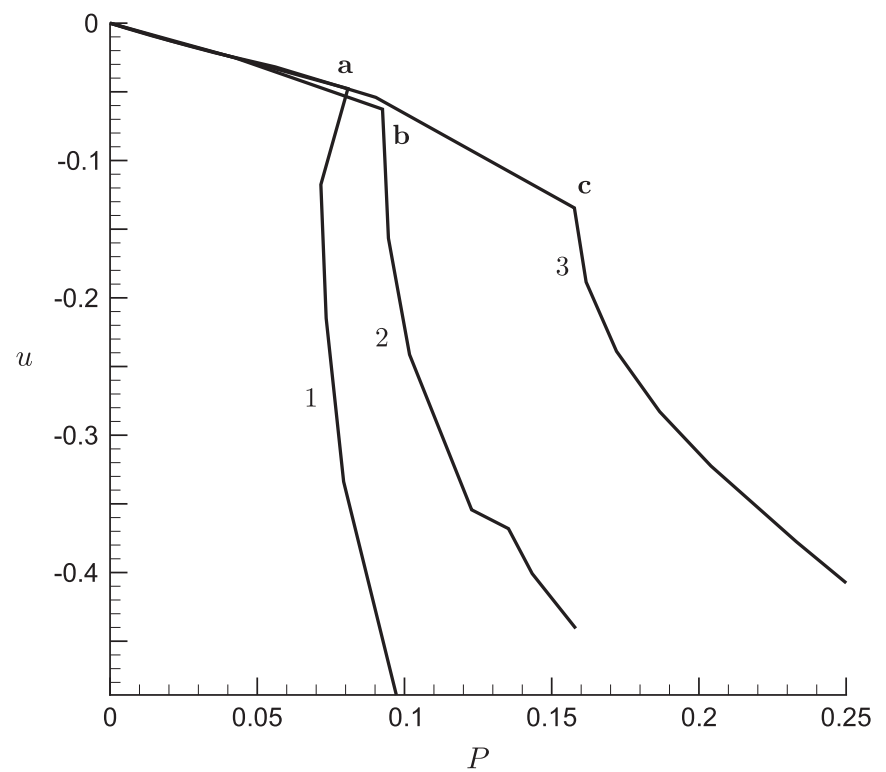

Fig. 17. Displacement component $u$ versus external pressure $P$ for the thinner and longer tube with $A / B=0.8, L / B=5$ at point $(X, Y, Z)=(0.73,0.67,0.17)$. The solution bifurcates at points $\mathbf{a}, \mathbf{b}, \mathbf{c}$ and into three different post-buckling modes ( $m=4, n=1,2,3$ ), labeled $1,2,3$, respectively.

cylindrical tubes with different aspect ratios. To valid our numerical code, comparisons have been made with corresponding linear models and with results from Abaqus.

In general, to produce higher mode bifurcations, greater external pressure is required. In this study we have not attempted to exhaust all possible solution branches. However, it is clear that the thinner and/or longer the tube, the more solution branches appear as the external pressure is increased. All of the collapsed deformations eventually lead to inner wall contacts, which are not considered here.

For thick-walled short tubes, only axisymmetric bifurcation modes are observed under either internal or external pressure. This indicates that the axisymmetric analysis provides a good approximation for thick-walled short tube problems. Thick-walled short tubes also exhibit complex corner bulging behavior which cannot be captured using the linear theory. Corner bulging is observed for the tubes under both internal and external pressures, and seems to be a strongly non-linear behavior caused by the end constraints.

For thick-walled longer tubes, our results show that the bifurcation pressure is the lowest for mode $(m=2, n=1)$, Fig. 12 , and the bifurcation is sub-critical. This agrees with previous observations of Wang and Ertepinar [51], Bertram [39] and Marzo et al. [19].

The critical bifurcation pressure for longer tubes is much smaller, and the post-buckling solution is no longer unique. In fact, depending on the initial parameters, the solution may follow different post-buckling paths. In addition, the difference in the critical pressures between neighboring bifurcation modes is very small, especially for thinner and longer tubes. In our numerical simulations, very small arc-length steps are needed to distinguish different bifurcation paths. We also found that these bifurcation states are sensitive to the incremental load applied. If the incremental load is large, the solution may jump on to a different post-buckling path. This is shown in Fig. 16(b). These deformations are not only highly non-linear, but also fully three-dimensional. The axisymmetric assumption can no longer be used here.

We make a particular observation on the compliance change of the longer tubes after the post-buckling path corresponding to $(m=2, n=1)$, i.e. the central circular cross-sectional area buckles in to an elliptical shape (see Fig. 19), and the transmural pressure (defined as internal minus external pressure, in this case $=-P$ ) is plotted against the ratio of the deformed to reference crosssectional area $A / A_{0}$ at $Z=0$ in Fig. 18. Clearly, after the point $\mathbf{d}$ in Fig. $12, A / A_{0}$ drops sharply against very small changes in $P$; in fact, there is a negative dependence on $-P$ for the thick-walled tube $(A / B=0.5, L / B=5)$, i.e. a smaller transmural pressure is required for the tube to collapse further, whereas for the thinner tube $(A / B=0.8, L / B=5)$ there is no negative dependence on $P$ after the initial buckling.

If we look at the first principal stress, then along path 1 the maximum stress jumps from the ends to the central cross-section $(Z=0)$ at the point $\mathbf{c}$ for the thick-walled tube, whereas for the thinner tube, the maximum stress remains at the ends (see Figs. 19 and 20). We believe the reason for the compliance change is due to the sudden collapse of the middle section of the thickerwalled tube. These tubes are more strongly constrained by the end boundaries and therefore have much stronger resistance to collapse until the external pressure is sufficiently large and the strain energy has accumulated to the point that the system snaps through to a lower energy state. The thinner-walled tubes, on the other hand, collapse more readily since there is no accumulation of strain energy as the external pressure increases further. Indeed, similar behavior is seen after the higher-mode bifurcations, as shown in Figs. 12 and 16(a) (paths 2 and 3).

This observation is interesting and has implications for collapsible tube problems since it has been reported both experimentally and numerically that in the post-buckling mode $(m=2, n=1)$ thick tubes can become more compliant than thinner ones [39,19]. This is supported by our results. Note that the lengths of the tubes used by Wang and Ertepinar [51], Bertram [39] and Marzo et al. [19] were much larger than those studied here, so that the end effects are less obvious. Clearly, the end constraints combined with wall thickness contribute to the sudden jumps (snap-through) behavior and negative transmural pressure dependence.

Finally, we recognize that Abaqus has superior solvers for the post-buckling analysis, and could be used to carry out all the simulations without using libMesh. In fact, the Abaqus solvers continue to give "solutions" even after the contact walls are penetrated unless supplemented by a contact model. We have not done

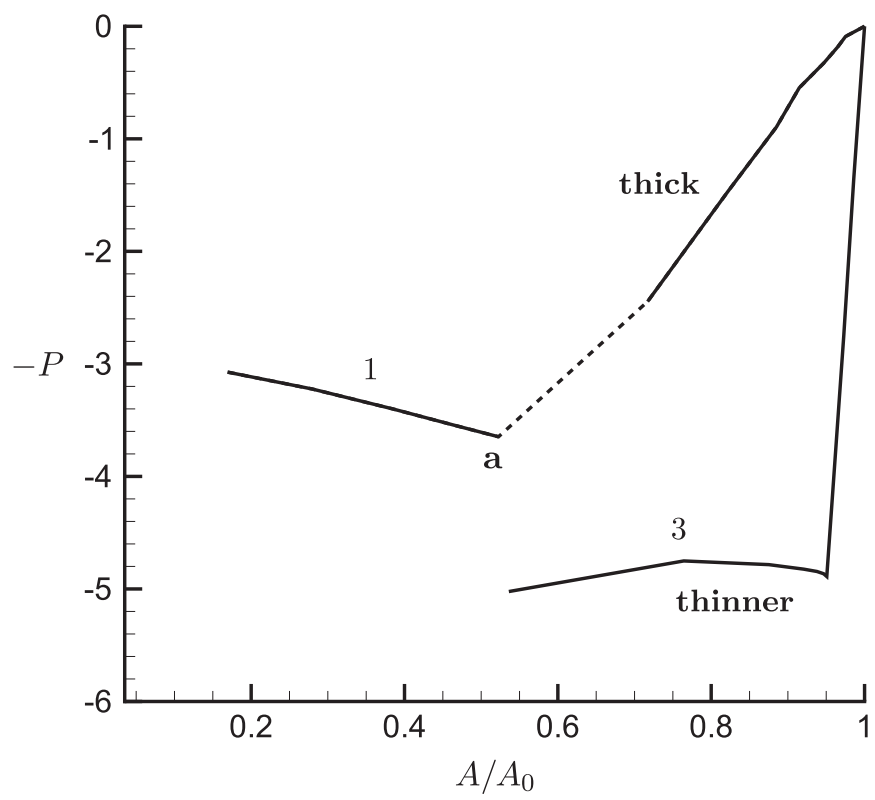

Fig. 18. The negative dependence of pressure on area is demonstrated by the plot of $(-P)$ versus cross-sectional area ratio $A / A_{0}$ following the post-buckling mode $(m=2, n=1)$ for the thick-walled longer tube, as in Fig. 12, and mode ( $m=5, n=1)$, for the thinner and longer tube, as in Fig. 16(a). See the text for detailed discussion. 

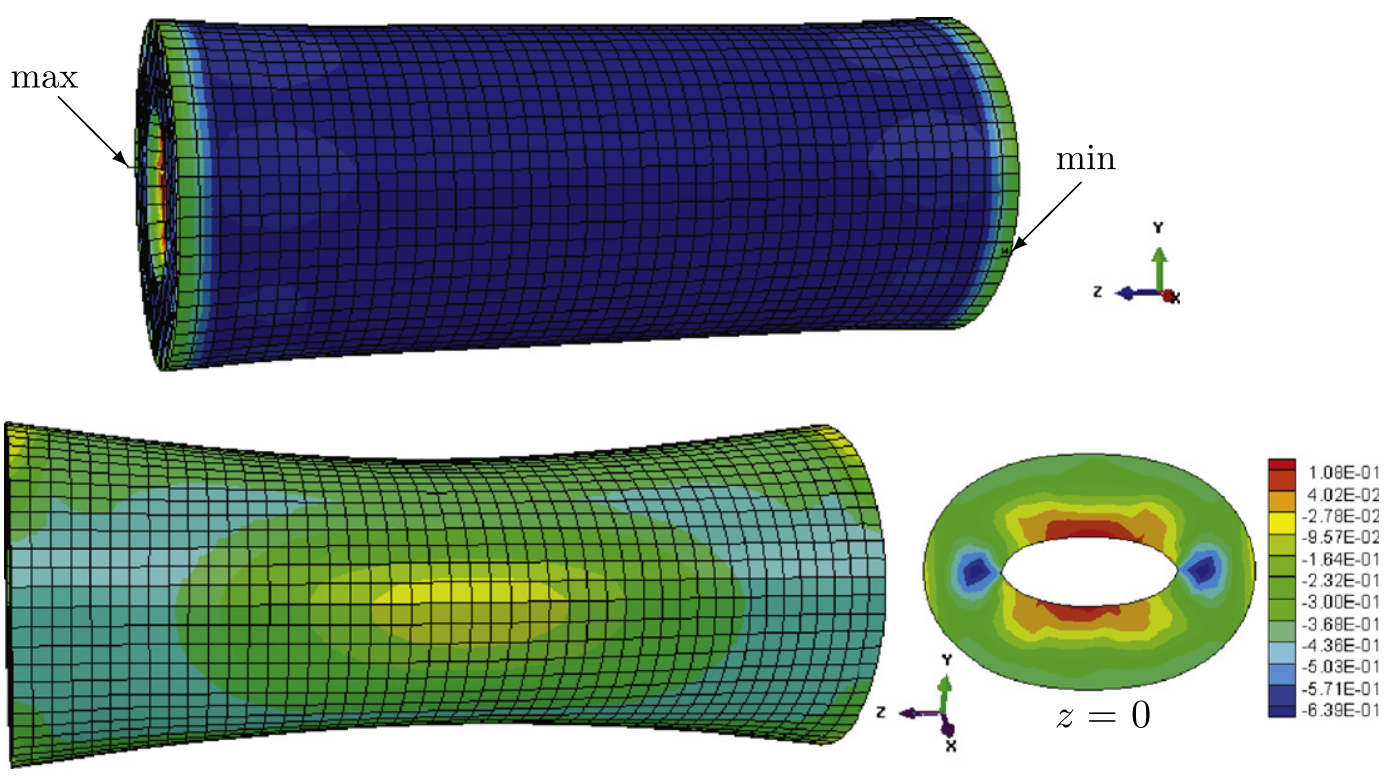

Fig. 19. Location of the maximum first principal stress changes following the post-buckling mode ( $m=2, n=1)$ before (top) and after (bottom) point $\mathbf{d}$ in Fig. 12 : thickwalled longer tube.
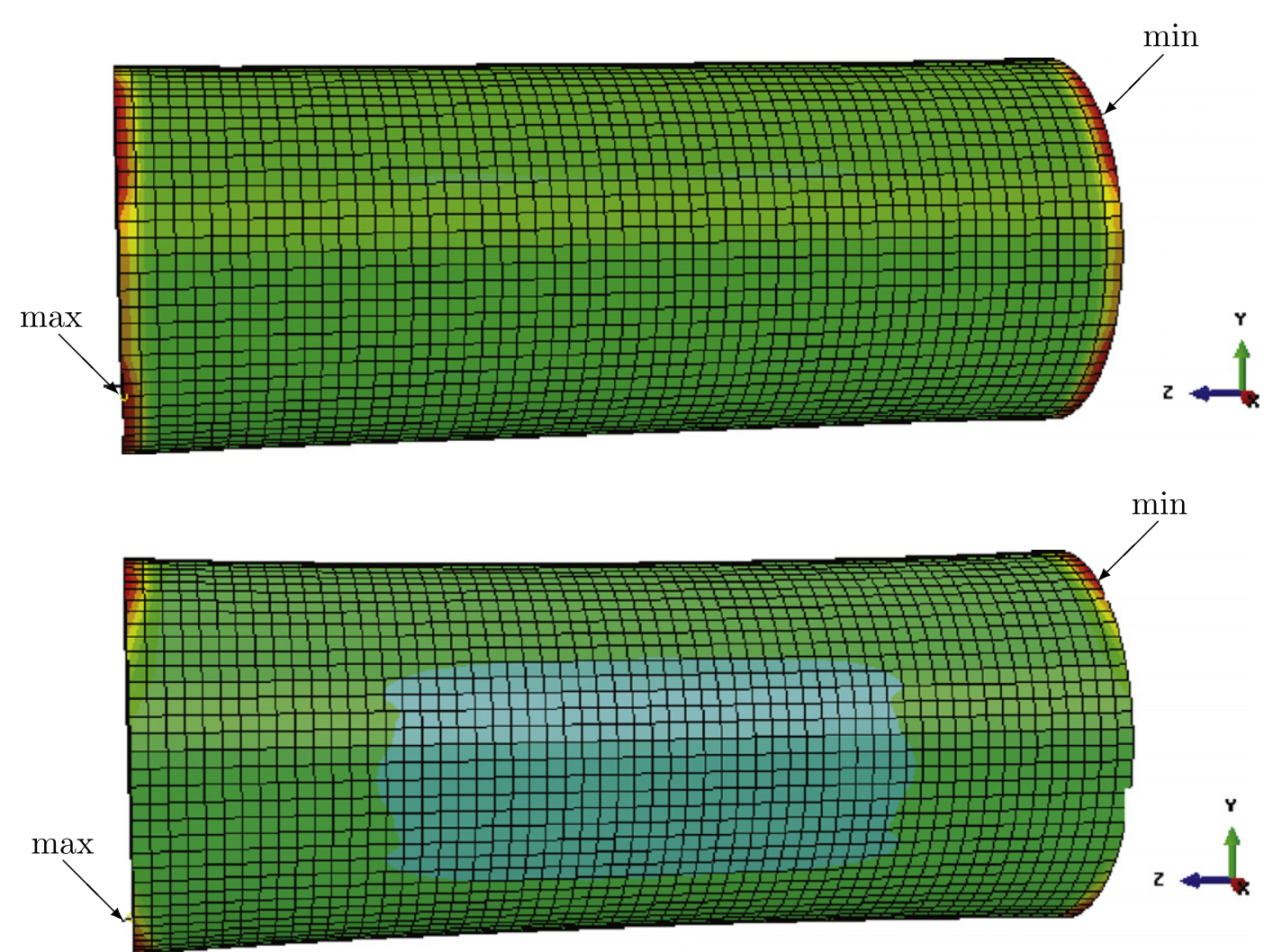

Fig. 20. Location of the maximum first principal stress changes following the post-buckling mode ( $m=2, n=1)$ before (top) and after (bottom) point a in Fig. 16: thinner and longer tube.

this since tube deformation after contact is not of interest in the present study. However, the purpose of developing our libMesh code is so that we have better control of numerical schemes, and can further develop the code for other physiological applications, such as heart modeling. In particular, we are interested in developing interfaces to fluid-structure interaction solvers such as immersed boundary codes. The human heart is also subject to internal pressure, and hence may not develop higher mode bifurcations in the normal physiological situation. In this respect, the current libMesh solvers are sufficient.

\section{Acknowledgments}

This work was sponsored by a Medical Research Scotland Grant. We are grateful to the Royal Academy of Engineering and 
the Royal Society of Edinburgh for travel funding. All simulations were performed on the Euclid workstations in the School of Mathematics and Statistics (libMesh code), and the Euler workstations in the School of Engineering (Abaqus code), University of Glasgow. X.Y.L. particularly wishes to thank Prof. C. Bertram at the University of New South Wales, Australia, who first observed the compliance change in thick-walled tubes in his experiments. The authors also wish to thank the libMesh developers, and Dr. B.E. Griffith at New York University, for useful discussions.

\section{References}

[1] W.A. Conrad, Pressure-flow relationships in collapsible tubes, IEEE Transactions on Biomedical Engineering 16 (1969) 284-295.

[2] A.I. Katz, Y. Chen, A.H. Moreno, Flow through a collapsible tube: experimental analysis and mathematical model, Biophysical Journal 9 (1969) 1261-1279.

[3] K. Ishizaka, J.L. Flanagan, Synthesis of voiced sounds from a two-mass model of the vocal cords, Bell System Technical Journal 51 (1972) 1233-1268.

[4] A.H. Shapiro, Steady flow in collapsible tubes, Journal of Biomechanical Engineering 99 (1977) 126-147.

[5] R.D. Kamm, A.H. Shapiro, Unsteady flow in a collapsible tube subjected to external pressure or body forces, The Journal of Fluid Mechanics 95 (1979) $1-78$.

[6] C.D. Bertram, T.J. Pedley, A mathematical model of unsteady collapsible tube behaviour, Journal of Biomechanics 15 (1982) 39-50.

[7] C. Cancelli, T.J. Pedley, A separated-flow model for collapsible-tube oscillations, The Journal of Fluid Mechanics 157 (1985) 375-404

[8] O.E. Jensen, Instabilities of flow in a collapsed tube, The Journal of Fluid Mechanics 220 (1990) 623-659.

[9] X.Y. Luo, T.J. Pedley, A numerical simulation of steady flow in a 2-D collapsible channel, Journal of Fluids and Structures 9 (1995) 149-174.

[10] X.Y. Luo, T.J. Pedley, A numerical simulation of unsteady flow in a twodimensional collapsible channel, The Journal of Fluid Mechanics 314 (1996) $191-225$.

[11] X.Y. Luo, T.J. Pedley, The effects of wall inertia on flow in a two-dimensional collapsible channel, The Journal of Fluid Mechanics 363 (1998) 253-280.

[12] X.Y. Luo, T.J. Pedley, Multiple solutions and flow limitation in collapsible channel flows, The Journal of Fluid Mechanics 420 (2000) 301-324.

[13] T.J. Pedley, X.Y. Luo, Modelling flow and oscillations in collapsible tubes, Theoretical and Computational Fluid Dynamics 10 (1998) 277-294.

[14] O.E. Jensen, M. Heil, High-frequency self-excited oscillations in a collapsiblechannel flow, The Journal of Fluid Mechanics 481 (2003) 235-268.

[15] X.Y. Luo, Z.X. Cai, W.G. Li, T.J. Pedley, The cascade structure of linear stabilities of flow in collapsible channels, The Journal of Fluid Mechanics 600 (2008) 45-76

[16] P.S. Stewart, M. Heil, S.L. Waters, O.E. Jensen, Sloshing and slamming oscillations in collapsible channel flow, The Journal of Fluid Mechanics 662 (2010) 288-319.

[17] H.F. Liu, X.Y. Luo, Z.X. Cai, Stability and energy budget of pressure-driven collapsible channel flows, Journal of Fluid Mechanics 705 (2012) 348-370.

[18] A.L. Hazel, M. Heil, Steady finite-Reynolds-number flows in three-dimensional collapsible tubes, The Journal of Fluid Mechanics 486 (2003) 79-103.

[19] A. Marzo, X.Y. Luo, C.D. Bertram, Three-dimensional collapse and steady flow in thick-walled flexible tubes, Journal of Fluids and Structures 20 (2005) 817-835.

[20] M. Heil, S.L. Waters, How rapidly oscillating collapsible tubes extract energy from a viscous mean flow, The Journal of Fluid Mechanics 601 (2008) 199-227.

[21] R.J. Whittaker, S.L. Waters, O.E. Jensen, J. Boyle, M. Heil, The energetics of flow through a rapidly oscillating tube. Part I: general theory, The Journal of Fluid Mechanics 648 (2010) 83-121.

[22] R.J. Whittaker, M. Heil, J. Boyle, O.E. Jensen, S.L. Waters, The energetics of flow through a rapidly oscillating tube. Part II: application to an elliptical tube, The Journal of Fluid Mechanics 648 (2010) 123-153.

[23] M. Heil, T.J. Pedley, Large post-buckling deformations of cylindrical shells conveying viscous flow, Journal of Fluids and Structures 10 (1996) 565-599.

[24] N. Yamaki, Buckling of circular cylindrical shells under external pressure, Reports of the Institute of High Speed Mechanics 20 (1969) 35-55.
[25] N. Yamaki, Elastic Stability of Circular Cylindrical Shells, North-Holland, Amsterdam, 1984

[26] M. Amabili, M.P. Païdoussis, Review of studies on geometrically nonlinear vibrations and dynamics of circular cylindrical shells and panels, with and without fluid-structure interaction, Applied Mechanics Reviews 56 (2003) 349-381.

[27] A.N. Kounadis, Recent advances on postbuckling analyses of thin-walled structures: beams, frames and cylindrical shells, Journal of Constructional Steel Research 62 (2006) 1101-1115.

[28] F.C. Bardi, S. Kyriakides, Plastic buckling of circular tubes under axial compression-Part I: experiments, International Journal of Mechanica Sciences 48 (2006) 830-841.

[29] F.C. Bardi, S. Kyriakides, H.D. Yun, Plastic buckling of circular tubes under axial compression-Part II: analysis, International Journal of Mechanical Sciences 48 (2006) 842-854.

[30] W.T. Koiter, Purpose and achievements of research in elastic stability, in: Recent Advances in Engineering Sciences, Proceedings of the 4th Technical Conference of the Society for Engineering Science, Raleigh, NC, 1966, pp. 197-218.

[31] W.T. Koiter, Elastic stability, buckling and post-buckling behaviour, in Proceedings of the IUTAM Symposium on Finite Elasticity, Lehigh University, 1980, 1981, pp. 13-24.

[32] G.J. Simitses, Buckling of moderately thick laminated cylindrical shells: a review, Composites B: Engineering 27 (1996) 581-587.

[33] J.W. Hutchinson, W.T. Koiter, Postbuckling theory, Applied Mechanics Reviews 23 (1974) 1353-1366.

[34] Y. Zhu, X.Y. Luo, R.W. Ogden, Asymmetric bifurcations of thick-walled circular cylindrical elastic tubes under axial loading and external pressure, International Journal of Solids and Structures 45 (2008) 3410-3429.

[35] J. Chróścielewski, W. Pietraszkiewicz, W. Witkowski, On shear correction factors in the non-linear theory of elastic shells, International Journal of Solids and Structures 47 (2010) 3537-3545.

[36] A. Libai, J.G. Simmonds, The Nonlinear Theory of Elastic Shells, second ed. Cambridge University Press, Cambridge, UK, 1998.

[37] Y. Goto, C. Zhang, Plastic buckling transition modes in moderately thick cylindrical shells, Journal of Engineering Mechanics 125 (1999) 426-434.

[38] C.D. Bertram, Two modes of instability in a thick-walled collapsible tube conveying a flow, Journal of Biomechanics 15 (1982) 223-224.

[39] C.D. Bertram, The effects of wall thickness, axial strain and end proximity on the pressure-area relation of collapsible tubes, Journal of Biomechanics 20 (1987) 863-876.

[40] C.D. Bertram, C.J. Raymond, T.J. Pedley, Mapping of instabilities for flow through collapsed tubes of differing length, Journal of Fluids and Structures 4 (1990) 125-153.

[41] C.D. Bertram, N.S.J. Elliott, Flow-rate limitation in a uniform thin-walled collapsible tube, with comparison to a uniform thick-walled tube and a tube of tapering thickness, Journal of Fluids and Structures 17 (2003) 541-559.

[42] J.L. Nowinski, M. Shahinpoor, Stability of an elastic circular tube of arbitrary wall thickness subjected to an external pressure, International Journal of Non-Linear Mechanics 4 (1969) 143-158.

[43] Y. Zhu, X.Y. Luo, R.W. Ogden, Nonlinear axisymmetric deformations of an elastic tube under external pressure, European Journal of Mechanics A/Solids 29 (2010) 216-229.

[44] B. Kirk, J.W. Peterson, R.H. Stogner, G.F. Carey, LibMesh: a C++ library for parallel adaptive mesh refinement/coarsening simulations, Engineering with Computers 22 (2006) 237-254.

[45] Abaqus 6.10 Documentation, 2010.

[46] E. Riks, An incremental approach to the solution of snapping and buckling problems, International Journal of Solids and Structures 15 (1979) 529-551.

[47] M.A. Crisfield, A fast incremental/iterative solution procedure that handles "snap-through", Computers \& Structures 13 (1981) 55-62.

[48] R.W. Ogden, Non-linear Elastic Deformations, Dover Publications, New York, 1997.

[49] S. Balay, K. Buschelman, V. Eijkhout, W.D. Gropp, D. Kaushik, M.G. Knepley, L.C. McInnes, B.F. Smith, H. Zhang, PETSc Users Manual. Technical Report ANL-95/11-Revision 3.0.0, Argonne National Laboratory, 2008.

[50] O.C. Zienkiewicz, J.Z. Zhu, The superconvergent patch recovery and a posteriori error estimates. Part 1: the recovery technique, International Journal of Numerical Methods in Engineering 33 (1992) 1331-1364.

[51] A.S.D. Wang, A. Ertepinar, Stability and vibrations of elastic thick-walled cylindrical and spherical shells subjected to pressure, International Journal of Non-Linear Mechanics 7 (1972) 539-555. 